\title{
Surveillance for Violent Deaths - National Violent Death Reporting System, 18 States, 2014
}




\section{CONTENTS}

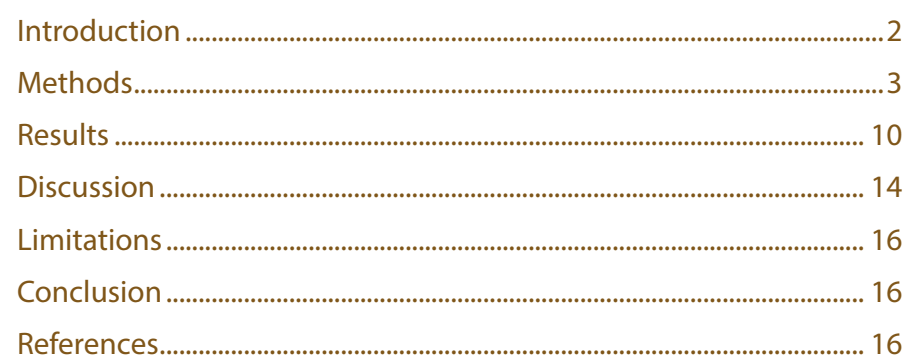

The MMWR series of publications is published by the Center for Surveillance, Epidemiology, and Laboratory Services, Centers for Disease Control and Prevention (CDC), U.S. Department of Health and Human Services, Atlanta, GA 30329-4027.

Suggested citation: [Author names; first three, then et al., if more than six.] [Title]. MMWR Surveill Summ 2018;67(No. SS-\#):[inclusive page numbers].

\section{Centers for Disease Control and Prevention}

Brenda Fitzgerald, MD, Director

Leslie Dauphin, PhD, Acting Associate Director for Science

Joanne Cono, MD, ScM, Director, Office of Science Quality

Chesley L. Richards, MD, MPH, Deputy Director for Public Health Scientific Services

Michael F. Iademarco, MD, MPH, Director, Center for Surveillance, Epidemiology, and Laboratory Services

\section{MMWR Editorial and Production Staff (Serials)}

Sonja A. Rasmussen, MD, MS, Editor-in-Chief

Charlotte K. Kent, PhD, MPH, Executive Editor

Christine G. Casey, MD, Editor

Mary Dott, MD, MPH, Online Editor

Teresa F. Rutledge, Managing Editor

David C. Johnson, Lead Technical Writer-Editor

Glenn Damon, Project Editor

Timothy F. Jones, MD, Chairman

Matthew L. Boulton, MD, MPH

Virginia A. Caine, MD

Katherine Lyon Daniel, PhD

Jonathan E. Fielding, MD, MPH, MBA

David W. Fleming, MD

Martha F. Boyd, Lead Visual Information Specialist Maureen A. Leahy, Julia C. Martinroe, Stephen R. Spriggs, Tong Yang, Visual Information Specialists Quang M. Doan, MBA, Phyllis H. King,

Paul D. Maitland, Terraye M. Starr, Moua Yang, Information Technology Specialists

MMWR Editorial Board

William E. Halperin, MD, DrPH, MPH

King K. Holmes, MD, PhD

Robin Ikeda, MD, MPH

Rima F. Khabbaz, MD

Phyllis Meadows, PhD, MSN, RN

Jewel Mullen, MD, MPH, MPA
Jeff Niederdeppe, $\mathrm{PhD}$

Patricia Quinlisk, MD, MPH

Patrick L. Remington, MD, MPH Carlos Roig, MS, MA

William L. Roper, MD, MPH

William Schaffner, MD 


\title{
Surveillance for Violent Deaths - National Violent Death Reporting System, 18 States, 2014
}

\author{
Katherine A. Fowler, $\mathrm{PhD}^{1}$ \\ Shane P.D. Jack, $\mathrm{PhD}^{1}$ \\ Bridget $\mathrm{H}$. Lyons, $\mathrm{MPH}^{1}$ \\ Carter J. Betz, MS ${ }^{1}$ \\ Emiko Petrosky, $\mathrm{MD}^{1}$ \\ ${ }^{1}$ Division of Violence Prevention, National Center for Injury Prevention and Control, CDC, Atlanta, Georgia
}

\begin{abstract}
Problem/Condition: In 2014, approximately 59,000 persons died in the United States as a result of violence-related injuries. This report summarizes data from CDC's National Violent Death Reporting System (NVDRS) regarding violent deaths from 18 U.S. states for 2014. Results are reported by sex, age group, race/ethnicity, marital status, location of injury, method of injury, circumstances of injury, and other selected characteristics.
\end{abstract}

Reporting Period Covered: 2014.

Description of System: NVDRS collects data from participating states regarding violent deaths. Data are obtained from death certificates, coroner/medical examiner reports, law enforcement reports, and secondary sources (e.g., child fatality review team data, supplemental homicide reports, hospital data, and crime laboratory data). This report includes data from 18 states that collected statewide data for 2014 (Alaska, Colorado, Georgia, Kentucky, Maryland, Massachusetts, Michigan, New Jersey, New Mexico, North Carolina, Ohio, Oklahoma, Oregon, Rhode Island, South Carolina, Utah, Virginia, and Wisconsin). NVDRS collates documents for each death and links deaths that are related (e.g., multiple homicides, a homicide followed by a suicide, or multiple suicides) into a single incident.

Results: For 2014, a total of 22,098 fatal incidents involving 22,618 deaths were captured by NVDRS in the 18 states included in this report. The majority of deaths were suicides (65.6\%), followed by homicides (22.5\%), deaths of undetermined intent (10.0\%), deaths involving legal intervention (1.3\%) (i.e., deaths caused by law enforcement and other persons with legal authority to use deadly force, excluding legal executions), and unintentional firearm deaths $(<1 \%)$. The term "legal intervention" is a classification incorporated into the International Classification of Diseases, Tenth Revision (ICD-10) and does not denote the lawfulness or legality of the circumstances surrounding a death caused by law enforcement. Suicides occurred at higher rates among males, non-Hispanic American Indian/Alaska Natives (AI/AN), non-Hispanic whites, persons aged 45-54 years, and males aged $\geq 75$ years. Suicides were preceded primarily by a mental health, intimate partner, substance abuse, or physical health problem or a crisis during the previous or upcoming 2 weeks. Homicide rates were higher among males and persons aged $<1$ year and 15-44 years; rates were highest among non-Hispanic black and AI/AN males. Homicides primarily were precipitated by arguments and interpersonal conflicts, occurrence in conjunction with another crime, or related to intimate partner violence (particularly for females). When the relationship between a homicide victim and a suspected perpetrator was known, it was most often either an acquaintance/ friend or an intimate partner. Legal intervention death rates were highest among males and persons aged 20-44 years; rates were highest among non-Hispanic black males and Hispanic males. Precipitating factors for the majority of legal intervention deaths were alleged criminal activity in progress, the victim reportedly using a weapon in the incident, a mental health or substance abuse problem, an argument or conflict, or a recent crisis. Deaths of undetermined intent occurred more frequently among males, particularly non-Hispanic black and AI/AN males, and persons aged 30-54 years. Substance abuse, mental health problems, physical health problems, and a recent crisis were the most common circumstances preceding deaths of undetermined intent. Unintentional firearm deaths were more frequent among males, non-Hispanic whites, and persons aged 10-24 years; these deaths most often occurred while the shooter was playing with a firearm and were most often precipitated by a person unintentionally pulling the trigger or mistakenly thinking the firearm was unloaded.

Corresponding author: Katherine A. Fowler, Division of Violence Prevention, National Center for Injury Prevention and Control, CDC. Telephone: 770-488-3916; E-mail: kafowler@cdc.gov.
Interpretation: This report provides a detailed summary of data from NVDRS for 2014. The results indicate that violent deaths resulting from self-inflicted or interpersonal violence disproportionately affected persons aged $<65$ years, males, and certain minority populations. The primary precipitating 
factors for homicides and suicides were intimate partner problems, interpersonal conflicts, mental health and substance abuse problems, and recent crises.

Public Health Action: NVDRS data are used to monitor the occurrence of violence-related fatal injuries and assist public health authorities in the development, implementation, and evaluation of programs and policies to reduce and prevent violent deaths. For example, North Carolina VDRS data were used to improve case ascertainment of pregnancy-associated suicides, Wisconsin VDRS data were used to develop the statewide suicide prevention strategy, and Colorado VDRS data were used to develop programs and prevention strategies for suicide among veterans. The continued development and expansion of NVDRS to include all U.S. states, territories, and the District of Columbia are essential to public health efforts to reduce the impact of violence.

\section{Introduction}

In 2014, approximately 59,000 persons died in the United States as a result of violence-related injuries (1). Suicide was the 10th leading cause of death overall in the United States and disproportionately affected young and middleaged populations. It was among the top two leading causes of death for persons aged 10-34 years and among the top four for persons aged 35-54 years. Non-Hispanic American Indian/Alaska Native and non-Hispanic white males were disproportionally affected by suicide.

Homicide was the 17th leading cause of death overall in the United States and disproportionately affected young persons (1). It was the third leading cause of death for persons aged 15-34 years, the fourth leading cause of death for children aged 1-9 years, and the fifth leading cause of death for persons aged 10-14 years. Homicide disproportionately affected young black males; it was the leading cause of death among nonHispanic black males aged 15-34 years.

Public health authorities require accurate, timely, and comprehensive surveillance data to better understand and ultimately prevent the occurrence of violent deaths in the United States (2). In 2000, in response to an Institute of Medicine* report noting the need for a national fatal intentional injury surveillance system (3), CDC began planning to implement the National Violent Death Reporting System (NVDRS) (2). The goals of NVDRS are to:

- collect and analyze timely, high-quality data for monitoring the magnitude and characteristics of violent deaths at national, state, and local levels;

- ensure data are disseminated routinely and expeditiously to public health officials, law enforcement officials, policymakers, and the public;

- ensure data are used to develop, implement, and evaluate programs and strategies that are intended to reduce and prevent violent deaths and injuries at national, state, and local levels; and

\footnotetext{
*The name of the Institute of Medicine was changed to the National Academy of Medicine, effective July 1, 2015.
}

- build and strengthen partnerships among organizations and communities at national, state, and local levels to ensure that data are collected and used to reduce and prevent violent deaths and injuries.

NVDRS is a state-based active surveillance system that collects data on the characteristics and circumstances associated with all violence-related deaths in participating states. Data on deaths include homicides, suicides, legal intervention deaths (i.e., deaths caused by law enforcement acting in the line of duty and other persons with legal authority to use deadly force but excluding legal executions), unintentional firearm deaths, and deaths of undetermined intent. ${ }^{\dagger}$ The term "legal intervention" is a classification incorporated into the International Classification of Diseases, Tenth Revision (ICD-10) and does not denote the lawfulness or legality of the circumstances surrounding a death caused by law enforcement. NVDRS data are used to assist the development, implementation, and evaluation of programs and strategies designed to reduce and prevent these deaths at the national, state, and local levels.

Before implementation of NVDRS, single data sources (e.g., death certificates or law enforcement reports) provided only limited information and few circumstances from which to understand patterns of violent deaths. NVDRS fills this surveillance gap by providing more detailed information. It is the first system to 1) provide detailed information on circumstances precipitating violent deaths, 2) link multiple source documents on violent deaths so that each incident can contribute to the study of patterns of violent deaths, and 3) link multiple deaths that are related to one another (e.g., multiple homicides, suicide pacts, or homicide followed by the suicide of the suspected perpetrator).

NVDRS began data collection in 2003 with six states (Maryland, Massachusetts, New Jersey, Oregon, South Carolina, and Virginia). Seven states (Alaska, Colorado,

\footnotetext{
${ }^{\dagger}$ To be included in NVDRS, deaths of undetermined intent must have some evidence of the possibility that the intent was purposeful, including use of a weapon or other evidence that force was used to inflict the injury. Most commonly, the coroner/medical examiner is unsure whether the death was a suicide or unintentional.
} 
Georgia, North Carolina, Oklahoma, Rhode Island, and Wisconsin) joined in 2004; four (California, Kentucky, New Mexico, and Utah) joined in 2005; and two (Ohio and Michigan) joined in 2010 (Figure). CDC provides funding for state participation, and the ultimate goal is for NVDRS to expand to include all 50 states, U.S. territories, and the District of Columbia. $\$$

This report summarizes data for 2014 for deaths meeting NVDRS inclusion criteria from the 18 states that collected statewide data in that year (Alaska, Colorado, Georgia, Kentucky, Maryland, Massachusetts, Michigan, New Jersey, New Mexico, North Carolina, Ohio, Oklahoma, Oregon, Rhode Island, South Carolina, Utah, Virginia, and Wisconsin); these states account for approximately $33.4 \%$ of the U.S. population $(1,4)$. The analysis in this report does not include data from California, which concluded its participation in 2009. NVDRS data are updated annually and are publicly available through CDC's Web-based Injury Statistics Query and Reporting System (WISQARS) at https://www.cdc. gov/injury/wisqars/nvdrs.html. Case-level NVDRS data are available to applicants who meet eligibility requirements via access to the NVDRS Restricted Access Database (https://www. cdc.gov/ViolencePrevention/NVDRS/RAD.html).

\section{Methods}

NVDRS compiles information from multiple data sources. The core required data sources are death certificates, coroner/ medical examiner reports, and law enforcement reports. Some participating states also collect information from secondary sources (e.g., child fatality review team data, supplemental homicide reports, and crime laboratory data). NVDRS collates documents for each death and links deaths that are related (e.g., multiple homicides, a homicide followed by a suicide, or multiple suicides) into a single incident. The ability to analyze linked data permits comprehensive assessment of violent deaths. This report presents select data for 2014. Additional data from 2014 are available at https://stacks.cdc.gov/view/cdc/50765.

In NVDRS, a violent death is defined as a death resulting from the intentional use of physical force or power, threatened or

\footnotetext{
\$In 2014, Arizona, Connecticut, Hawaii, Iowa, Illinois, Indiana, Kansas, Maine, Minnesota, New Hampshire, New York, Pennsylvania, Vermont, and Washington joined the system. In 2016, Alabama, California, Delaware, Louisiana, Missouri, Nebraska, Nevada, Puerto Rico, Washington, D.C., and West Virginia joined the system for a total of 42 states and territories participating. Fourteen of these states did not report data until 2015 and the other 10 states and territories did not report data until 2017.

S Frequencies and rates of violent deaths included in this report will differ slightly from the frequencies and rates of violent deaths reported by NVDRS WISQARS, which excludes nonresident deaths that occur in participating states (i.e., occurrent deaths). NVDRS tracks both resident and occurrent violent deaths in the overall data set, and the numbers in this report reflect both.
}

FIGURE. States participating in the National Violent Death Reporting System, by year of initial data collection, United States, 2003-2014

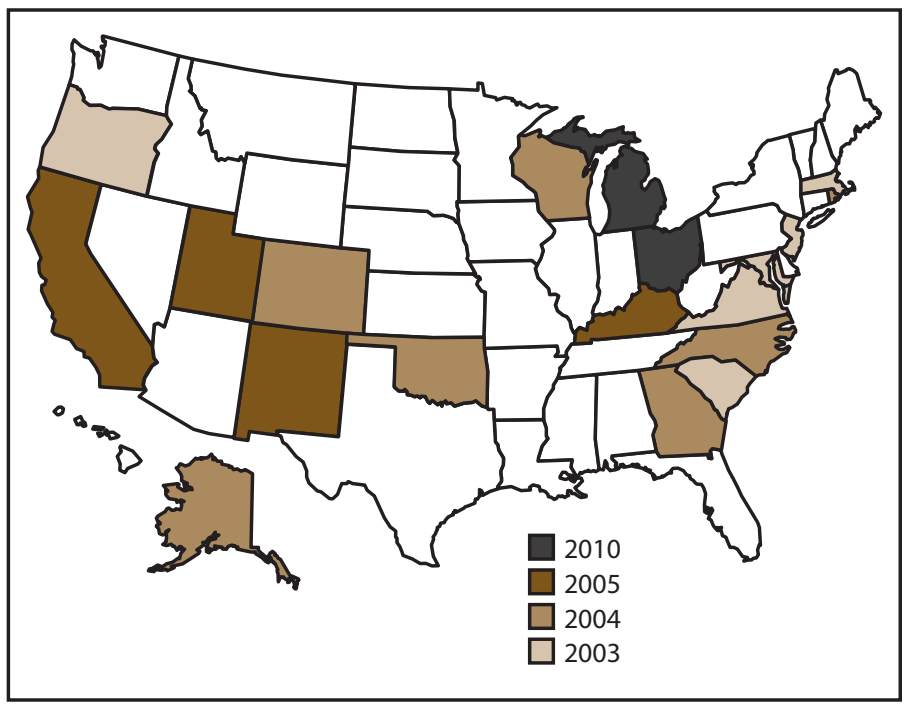

actual, against oneself, another person, or a group or community (5). Information also is collected about unintentional firearm deaths (i.e., a death resulting from a penetrating injury or gunshot wound from a weapon that uses a powder charge to fire a projectile when there was a preponderance of evidence that the shooting was not intentionally directed at the victim) and deaths of undetermined intent (i.e., a death that results from the use of force or power against oneself or another person for which the evidence indicating one manner of death is no more compelling than evidence indicating another). NVDRS cases are coded according to $I C D-10$ guidelines $(6)$ or on the basis of the manner of death assigned by the coroner/medical examiner or law enforcement. Cases are included if they are assigned $I C D-10$ codes (Box 1) or if the manner of death specified in at least one of the three primary data sources is consistent with NVDRS case definitions.

Variables analyzed in NVDRS include:

- manner of death (i.e., the intent [homicide/legal intervention, suicide, unintentional, undetermined] of the person inflicting a fatal injury);

- mechanism of injury (i.e., the method used to inflict a fatal injury) (Box 2);

- toxicology findings (for decedents who were tested);

- circumstances preceding injury (i.e., the events that preceded and were identified by investigators as relevant and therefore might have contributed to the infliction of a fatal injury) (Box 3);

- whether the decedent was a victim (i.e., a person who died as a result of a violence-related injury) or both a suspect and a victim (i.e., a person believed to have inflicted a fatal 
Surveillance Summaries

BOX 1. International Classification of Diseases, Tenth Revision (ICD-10) codes used in the National Violent Death Reporting System

\begin{tabular}{|lll|}
\hline Manner of death & Death $\leq \mathbf{1}$ year after injury & Death $>\mathbf{1}$ year after injury \\
\hline Intentional self-harm (suicide) & $\mathrm{X} 60-\mathrm{X} 84$ & Y87.0 \\
Assault (homicide) & $\mathrm{X} 85-\mathrm{X} 99, \mathrm{Y} 00-\mathrm{Y} 09$ & $\mathrm{Y} 87.1$ \\
Event of undetermined intent & $\mathrm{Y} 10-\mathrm{Y} 34$ & $\mathrm{Y} 87.2, \mathrm{Y} 89.9$ \\
Unintentional exposure to inanimate mechanical & $\mathrm{W} 32-\mathrm{W} 34$ & Y86 determined to be attributable \\
forces (firearms) & & to firearms \\
Legal intervention (excluding executions, Y35.5) & Y35.0-Y35.4, Y35.6-Y35.7 & Y89.0 \\
Terrorism & U01, U03 & U02 \\
\hline
\end{tabular}

injury on a victim who then was fatally injured [e.g., the perpetrator of a homicide-suicide]);

- information about any known suspects (i.e., a person believed to have inflicted a fatal injury on a victim);

- incident (i.e., an occurrence in which one or more persons sustained a fatal injury that was linked to a common event or perpetrated by the same suspect during a 24-hour period); and

- type of incident (i.e., a combination of the manner of death and the number of victims in an incident).

NVDRS is an incident-based system, and all decedents associated with a given incident are grouped in one record. Decisions about whether two or more deaths are related and belong to the same incident are made on the basis of the timing of the injuries rather than on the timing of the deaths. Deaths resulting from injuries that occur within 24 hours of each other (i.e., the 24-hour rule) and are clearly linked by source documents (discussed under Manner of Death) would be considered part of the same incident. Examples of an incident include 1) a single isolated violent death, 2) two or more related homicides (including legal intervention deaths) when the fatal injuries were inflicted $<24$ hours apart, 3) two or more related suicides or deaths of undetermined intent when the fatal injuries were inflicted $<24$ hours apart, and 4) a homicide followed by a suicide when both fatal injuries were inflicted $<24$ hours apart ( 7 ).

Data collected from individual information sources are entered into the NVDRS online data entry system (2). In 2013, NVDRS began using a streamlined coding system to facilitate data abstraction efficiency by eliminating the need to enter data into source-specific data entry screens. The streamlined interface allows data from multiple sources to be entered into the same incident record and includes internal validation checks, hover-over features that define selected fields, and other quality control measures. Primacy rules and hierarchal algorithms related to the source documents are now occurring at the state level. Access to the online system is provided to each state by CDC. State project personnel are provided ongoing coding training to help increase data quality. Data are transmitted continually through the web to a CDC-based server. No personally identifiable information is transmitted to CDC.

\section{Manner of Death}

A manner (i.e., intent) of death for each decedent is assigned by a trained abstractor who assimilates information from all source documents. The abstractor-assigned manner of death must agree with at least one core required data source; typically, all source documents are consistent regarding the manner of death. When there is a discrepancy, the abstractor must assign a manner of death on the basis of the preponderance of evidence in the source documents, but such occurrences are rare ( 7 ). For example, if two sources report a death as a suicide and a third reports it as a death of undetermined intent, the death is coded as a suicide.

NVDRS data are categorized into five abstractor-assigned manners of death: 1) suicide, 2) homicide, 3) unintentional firearm, 4) undetermined intent, and 5) legal intervention.

- Suicide. Suicide is a death resulting from the use of force against oneself when a preponderance of evidence indicates that the use of force was intentional. This category also includes the following scenarios: 1) deaths of persons who intended only to injure rather than kill themselves; 2) persons who initially intended to kill themselves, changed their minds, but ultimately died as a result of the act; 3 ) deaths associated with risk-taking behavior without clear intent to inflict fatal self-injury but associated with high risk for death (e.g., playing Russian roulette); 4) suicide that occurred while under the influence of substances or drugs taken voluntarily; 5) suicide that occurred while under the influence of a mental illness; and 6) suicide involving another person providing only passive assistance to the decedent (e.g., supplying the means or information needed to complete the act). This category does not include deaths 
caused by chronic or acute substance abuse without the intent to die or deaths attributed to autoerotic behavior (e.g., selfstrangulation during sexual activity). Corresponding ICD-10 codes included in NVDRS are X60-X84 and Y87.0 (Box 1).

- Homicide. Homicide is a death resulting from the use of physical force or power, threatened or actual, against another person, group, or community when a preponderance of evidence indicates that the use of force was intentional. Two special scenarios that the National Center for Health Statistics (NCHS) regards as homicides are included in the NVDRS case definition: 1) arson with no specified intent to injure someone and 2) a stabbing with intent unspecified. This category also includes the following scenarios: 1) deaths when the suspect intended only to injure rather than kill the victim, 2) deaths resulting from heart attack induced when the suspect uses force or power against the victim, 3) deaths that occur when a person kills an attacker in self-defense, 4) deaths resulting from a weapon that discharges unintentionally while being used to control or frighten a victim, 5) deaths attributed to child abuse without intent being specified, 6) deaths attributed to an intentional act of neglect by one person against another, 7) death of a child after birth that results from a direct injury due to violence sustained before birth, 8) deaths identified as a justifiable homicide where person committing homicide was not a law enforcement officer, and 9) deaths resulting from an act of terrorism. This category excludes vehicular homicide without intent to injure, unintentional poisoning deaths due to illegal or prescription drug overdose even when the person who provided drugs was charged with homicide, unintentional firearm deaths (a separate category), combat deaths or acts of war, deaths of unborn fetuses, and deaths of children after birth that resulted indirectly from violence sustained by the mother before birth (e.g., death from prematurity following premature labor brought on by violence). Corresponding ICD-10 codes included in NVDRS are U01-U03, X85-X99, Y00-Y09, and Y87.1 (Box 1).

- Unintentional firearm. An unintentional firearm death is a death resulting from a penetrating injury or gunshot wound from a weapon that uses a powder charge to fire a projectile and for which a preponderance of evidence indicates that the shooting was not directed intentionally at the decedent. Examples include a person who dies as a result of celebratory firing that was not intended to frighten, control, or harm anyone; a person who unintentionally shoots himself when using a firearm to frighten, control, or harm another person; a soldier shot during a field exercise but not in a combat situation; a person who received a self-inflicted wound while playing with a firearm; a person who mistakenly believes a gun is unloaded and shoots another person; a child aged $<6$ years who shoots him- or herself or another person; and a child
BOX 2. Methods used to inflict injury - National Violent Death Reporting System, 18 states, 2014

- Firearm: method that uses a powder charge to fire a projectile

- Hanging/strangulation/suffocation: hanging by the neck, manual strangulation, or plastic bag over the head

- Poisoning: street drug, alcohol, pharmaceutical, carbon monoxide, gas, rat poison, or insecticide

- Sharp instrument: knife, razor, machete, or pointed instrument (e.g., chisel or broken glass)

- Blunt instrument: (e.g., club, bat, rock, or brick)

- Fall: being pushed or jumping

- Motor vehicle: (e.g., car, bus, motorcycle, or other transport vehicle)

- Personal weapons: (e.g., hands, fists, or feet)

- Drowning: inhalation of liquid in bathtub, lake, or other source of water/liquid

- Fire/burns: inhalation of smoke or the direct effects of fire or chemical burns

- Intentional neglect: starvation, lack of adequate supervision, or withholding of health care

- Other: any method other than those already listed

- Unknown: method not reported or not known

who dies after birth from an unintentional firearm injury that was sustained in utero. This category excludes firearm injuries caused by unintentionally striking a person with the firearm (e.g., hitting a person on the head with the firearm rather than firing a projectile) and unintentional injuries from nonpowder guns (e.g., BB, pellet, or other compressed air-powered or gas-powered guns). Corresponding ICD-10 codes included in NVDRS are W32-W34 and Y86 (Box 1).

- Undetermined intent. A death of undetermined intent is a death resulting from the use of force or power against oneself or another person for which the evidence indicating one manner of death is no more compelling than evidence indicating another. This category includes coroner/medical examiner rulings (e.g., accident or suicide, undetermined, jumped or fell, or self-inflicted injury) when records give no evidence or opinions in favor of either unintentional or intentional injury. Corresponding ICD-10 codes in NVDRS are Y10-Y34, Y87.2, and Y89.9 (Box 1).

- Legal intervention. A death from legal intervention is a death in which a person is killed or died as a result of a law enforcement officer or other peace officer (i.e., a person with specified legal authority to use deadly force), including 


\section{Suicide/Undetermined Intent}

- Intimate partner problem: decedent was experiencing problems with a current or former intimate partner.

- Suicide of friend or family: decedent was distraught over, or reacting to, the suicide of a friend or family member.

- Other death of friend or family: decedent was distraught over, or reacting to, the nonsuicide death of a friend or family member.

- Physical health problem: decedent was experiencing physical health problems (e.g., a recent cancer diagnosis or chronic pain).

- Job problem: decedent was either experiencing a problem at work or was having a problem with joblessness.

- Recent criminal legal problem: decedent was facing criminal legal problems.

- Noncriminal legal problem: decedent was facing civil legal problems (e.g., a child custody or civil lawsuit).

- Financial problem: decedent was experiencing problems such as bankruptcy, overwhelming debt, or foreclosure of a home or business.

- Eviction or loss of home: decedent was experiencing a recent eviction or other loss of housing.

- School problem: decedent was experiencing a problem such as poor grades, bullying, social exclusion at school, or performance pressures.

- Traumatic anniversary: the incident occurred on or near the anniversary of a traumatic event in the decedent's life.

- Exposure to disaster: decedent was exposed to a disaster (e.g., earthquake or bombing).

- Left a suicide note: decedent left a note, e-mail message, video, or other communication indicating intent to die by suicide.

- Disclosed intent to die by suicide: decedent had previously expressed suicidal feelings to another person with time for that person to intervene.

- History of suicide thoughts or plans: decedent had previously expressed suicidal thoughts or plans.

- History of suicide attempts: decedent had previously attempted suicide before the fatal incident.

military police, while on duty. The term "legal intervention" is a classification from $I C D-10(\mathrm{Y} 35.0)$ and does not denote the lawfulness or legality of the circumstances surrounding the death. Legal intervention deaths include a small subset in which force was applied without clear lethal intent (e.g., during restraint or when applying force with a typically nondeadly weapon such as a Taser [Taser International, Scottsdale, Arizona]) or in which the death occurred while the person was fleeing capture. This category excludes legal executions. Corresponding ICD-10 codes included in NVDRS are Y35.0Y35.4, Y35.6, Y35.7, and Y89.0 (Box 1).

\section{Variables Analyzed}

NVDRS collects approximately 600 unique variables for each death. The number of variables recorded for each incident depends on the content and completeness of the source documents. Variables include manner of death; demographic information; ICD-10 cause of death codes and text descriptors; location, date, and time of injury and death; toxicology results; bodily injuries; precipitating circumstances; victim-suspect relationship; and method of injury (Boxes 1-3).

\section{Circumstances Preceding Death}

The circumstances preceding death are defined as the precipitating events that contributed to the infliction of a fatal injury (Box 3). The circumstances are reported on the basis of the content of the coroner/medical examiner and law enforcement investigative reports. Some circumstances are coded to a specific manner of death (e.g., suicide or death of undetermined intent); other circumstances are coded across all manners of death. The data abstractor selects from a list of potential circumstances and is required to code all known 


\section{Homicide/Legal Intervention}

- Jealousy (lovers' triangle): jealousy or distress over an intimate partner's relationship or suspected relationship with another person.

- Stalking: pattern of unwanted harassing or threatening tactics by either the decedent or suspect.

- Prostitution: prostitution or related activity that includes prostitutes, pimps, clients, or others involved in such activity.

- Drug involvement: drug dealing, drug trade, or illegal drug use.

- Brawl: mutual physical fight involving three or more persons.

- Mercy killing: decedent wished to die because of terminal or hopeless disease or condition, and documentation indicates that the decedent wanted to be killed.

- Victim was a bystander: decedent was not the intended target in the incident (e.g., pedestrian walking past a gang fight).

- Victim was a police officer on duty: decedent was a law enforcement officer killed in the line of duty.

- Victim was an intervener assisting a crime victim: decedent was attempting to assist a crime victim at the time of the incident (e.g., child attempts to intervene and killed while trying to assist a parent who is being assaulted).

- Victim used a weapon: decedent used a weapon to attack or defend during the course of the incident.

- Intimate partner violence-related: incident is related to conflict between current or former intimate partners; includes the death of actual intimate partners and nonintimate partner victims killed to cause pain to an intimate partner (e.g., child or parent).

- Hate crime: decedent was intentionally selected because of his/her actual or perceived gender, religion, sexual orientation, race/ethnicity, or disability.

- Mentally ill suspect: suspect's attack on decedent believed to be the direct result of a mental illness.

- Drive-by shooting: suspect drove near the decedent and fired a weapon while driving.

- Walk-by assault: decedent was killed by a targeted attack (e.g., ambush) where the suspect fled on foot.

- Random violence: decedent was killed by a random act of violence.

- Gang-related: incident resulted from gang activity or gang rivalry; not used if the decedent was a gang member and the death did not appear to result from gang activity.

circumstances that relate to each incident. If circumstances are not known (e.g., for a body found in the woods with no other details reported), the data abstractor leaves the "circumstances known" variable blank; these deaths are excluded from the denominator for circumstance values. If either the coroner/ medical examiner record or the law enforcement report indicates the presence of a circumstance, the abstractor endorses the circumstance (e.g., if the law enforcement report indicated that a decedent had disclosed an intent to die by suicide, then suicidal intent is endorsed).

\section{Coding Training and Quality Control}

Ongoing coding support for data abstractors is provided through an e-mail help desk, monthly conference calls with all states, and regular conference calls with individual states. States also can conduct additional abstractor training workshops and activities at their own discretion. An NVDRS coding manual (7) with CDC-issued standard guidance on coding criteria and examples for each data element is provided. Software features to enhance coding reliability include automated validation rules and a hover-over feature containing variable-specific information. 


\section{All Manners of Death (except Unintentional Firearm)}

- Current depressed mood: decedent was perceived by self or others to be depressed.

- Current diagnosed mental health problem: decedent was identified as having a mental health disorder or syndrome listed in the Diagnostic and Statistical Manual, Version IV (DSM-IV), with the exception of alcohol and other substance dependence (these are captured in separate variables).

- Type of mental health diagnosis: identifies the DSM-IV diagnosis made by a medical or mental health practitioner.

- Current mental health treatment: decedent was currently receiving mental health treatment as evidenced by a current prescription for a psychotropic medication or visit to a mental health professional in the previous 2 months.

- History of treatment for mental health problem: decedent was identified as having ever received mental health treatment within the decedent's lifetime.

- Alcohol/other substance problem: decedent was perceived by self or others to have a problem with, or to be addicted to, alcohol or other drugs.

- Other addiction: decedent was perceived by self or others to have an addiction other than alcohol or other substance abuse (e.g., gambling or sexual).

- Family relationship problem: decedent was experiencing problems with a family member other than an intimate partner.

- Other relationship problem: decedent was experiencing problems with a friend or associate (other than an intimate partner or family member).

- History of child abuse/neglect: decedent had history of physical, sexual, or psychological abuse; physical, emotional, or educational neglect; or exposure to a violent environment or inadequate supervision by a caretaker as a child.

- Caretaker abuse/neglect led to death: decedent was experiencing physical, sexual, or psychological abuse; physical, emotional, or educational neglect; or exposure to a violent environment or inadequate supervision by a caretaker that led to death.

- Perpetrator of interpersonal violence in previous month: decedent perpetrated interpersonal violence during the previous month.

- Victim of interpersonal violence in previous month: decedent was the target of interpersonal violence in the past month.

- Physical fight: a physical fight between two individuals which resulted in the death of the decedent who was either involved in the fight, a bystander, or trying to stop the fight.

- Argument or conflict: a specific argument or disagreement occurred during the incident.

- Precipitated by another crime: incident occurred as the result of another serious crime.

- Nature of crime: identifies the specific type of other crime that occurred during incident (e.g., robbery or drug trafficking).

- Crime in progress: serious crime was in progress at the time of the incident.

- Terrorist attack: decedent was injured in a terrorist attack, leading to death.

- Crisis during previous or upcoming 2 weeks: circumstance that is a current crisis or acute precipitating event that had either occurred in the previous 2 weeks or was impending in the following 2 weeks (e.g., a trial for a criminal offense begins the following week).

- Other crisis: A crisis related to a death but not captured by any of the circumstances. 


\section{Unintentional Firearm Death \\ Context of Injury}

- Hunting: death occurred any time after leaving home for a hunting trip and before returning home from a hunting trip.

- Target shooting: shooter was aiming for a target and unintentionally hit the decedent; can be at a shooting range or an informal backyard setting (e.g., teenagers shooting at signposts on a fence).

- Loading/unloading gun: gun discharged when the shooter was loading/unloading ammunition.

- Cleaning gun: shooter pulled trigger or gun discharged while cleaning, repairing, assembling, or disassembling gun.

- Showing gun to others: showing the gun to another person when the gun discharged or the trigger was pulled.

- Playing with gun: shooter and one or more others were playing with a gun when it discharged.

- Celebratory firing: shooter fired gun in celebratory manner (e.g., on New Year's Eve).

- Other context of injury: shooting occurred during some context other than those already described.

\section{Mechanism of Injury}

- Unintentionally pulled trigger: shooter unintentionally pulled the trigger (e.g., while grabbing the gun or holding it too tightly).

- Thought gun safety was engaged: shooter thought the safety was on and gun would not discharge.

- Thought unloaded/magazine disengaged: shooter thought the gun was unloaded because the magazine was disengaged.

- Thought gun was unloaded: shooter thought the gun was unloaded for other unspecified reason.

- Bullet ricochet: bullet ricocheted from its intended target and unintentionally struck the decedent.

- Gun defect or malfunction: gun had a defect or malfunctioned, as determined by a trained firearm examiner.

- Gun fired while holstering: gun was being replaced or removed from holster/clothing.

- Dropped gun: gun discharged when it was dropped or when something was dropped on it.

- Gun fired while operating safety/lock: shooter unintentionally fired the gun while operating the safety lock.

- Gun mistaken for toy: gun was mistaken for a toy and was fired without the user understanding the danger.

- Other mechanism of injury: shooting occurred as the result of a mechanism not already described.

States are asked to perform annual blind reabstractions of a subset of cases using multiple abstractors to identify inconsistencies. CDC also conducts a quality control analysis in which multiple variables are reviewed for their appropriateness, with special focus on abstractor-assigned variables (e.g., method and manner of death). If CDC finds inconsistencies, the state is notified and asked for a response or correction.

\section{Time Frame}

States are required to report all deaths within 6 months of the end of each calendar year for the preceding January-December.
States then have an additional 12 months to complete each incident record. Although states typically meet these requirements, additional details sometimes arrive after a deadline has passed. New incidents also might be identified after the deadline (e.g., a death certificate is revised, new evidence is obtained that changes a manner of death, or an ICD-10 miscoding is corrected to meet NVDRS inclusion criteria). These additional data are incorporated into NVDRS. Analysis files are updated in real time in the online system. CDC estimates that case counts might increase 1.0\%-2.0\% after the 18-month data collection period. 


\section{Fatal Injuries in 2014}

This report provides preliminary data concerning fatal injuries meeting the NVDRS case definition for violent deaths in 2014 that were received by CDC as of August 9, 2016 for 18 participating states. Participating states used vital statistics death certificate files or coroner/medical examiner reports to identify violent deaths meeting NVDRS case definitions. Each state reported all violent deaths of residents that occurred within the state and those of nonresidents for whom a fatal injury occurred within the state (i.e., occurrent deaths). Once a violent death was identified, NVDRS data abstractors linked source documents, linked deaths within each incident, coded data elements, and wrote short narratives of the incident. State-level data were then consolidated and analyzed for this aggregate report.

Numbers, percentages, and crude rates are presented in aggregate for all deaths by abstractor-assigned manner of death. Rates for cells with frequency $<20$ are not reported because of the instability of those rates (8). Rates could not be calculated for some variables (e.g., marital status and precipitating circumstances) because denominators were unknown. Bridgedrace 2014 population estimates were used as denominators in the crude rate calculations (9). For compatible numerators for rate calculations to be derived, records listing multiple races were recoded to a single race, when possible, using racebridging methods described by NCHS (https://www.cdc.gov/ nchs/nvss/bridged_race.htm).

\section{Results}

\section{All Deaths Captured by NVDRS}

\section{Deaths by Manner, Method, and Location}

The 18 NVDRS states included in this report collected data concerning 22,098 incidents and 22,618 deaths that occurred in 2014. The crude death rate was 21.2 deaths per 100,000 population. Suicides $(n=14,834$ [65.6\%]) accounted for the highest rate of violent deaths (13.9 per 100,000 population), followed by homicides $(\mathrm{n}=5,100$ [22.5\%]) (4.8 per 100,000 population). Deaths of undetermined intent $(\mathrm{n}=2,257$ [10.0\%]), legal intervention deaths $(\mathrm{n}=283[1.3 \%])$, and unintentional firearm deaths $(\mathrm{n}=144[<1.0 \%])$ occurred at lower rates $(2.1,0.3$, and 0.1 per 100,000 population, respectively). Firearms were the method used in $51.1 \%$ of deaths, hanging/strangulation/suffocation in $18.8 \%$, and poisoning in $17.4 \%$ (rates: $10.8,4.0$, and 3.7 per 100,000 population, respectively). Rates for all other methods were lower. For all deaths, a house or apartment was the most common location where injury occurred (69.5\%), followed by a street or highway (6.3\%), a motor vehicle (4.9\%), or a natural area $(4.0 \%)$ (Table 1$)$.

\section{Substance Use}

Poisoning and substance abuse figure prominently in violent deaths. In 2014, poisoning was the third most common means of suicide captured by NVDRS overall and was the top suicide method for women. In addition, poisoning deaths constitute the overwhelming majority of deaths of undetermined intent captured by NVDRS (73.8\%).

Approximately $75 \%$ of all decedents who received toxicology testing had one or more substances detected, and $42.8 \%$ had two or more (range: two-12). These percentages were even higher for decedents with a history of drug or alcohol abuse (i.e., substance abuse): of those decedents, $87.8 \%$ had one or more substances detected in their system at the time of death, and $53.3 \%$ had more than one.

The percentage of suicide decedents with prior substance abuse problems (other than alcohol) who died by poisoning was twice as high as the percentage of suicide decedents without prior substance abuse problems who died by poisoning (26\% versus $13 \%$, respectively). The most common substance types detected in suicide decedents with a history of substance abuse were alcohol $(51.1 \%)$, over-the-counter or otherwise unclassified drugs $(31.7 \%)$, benzodiazepines (23.3\%), opioids (23.2\%), antidepressants (20\%), and marijuana (13.6\%).

Toxicology results also reveal the substances that were not only present in the systems of decedents across all manners but also identify those that directly caused the person's death. The substances that caused the largest percentage of poisoning deaths across all manners of deaths were opioids $(38 \%)$, over-the-counter or otherwise unclassified drugs (29\%), benzodiazepines (19\%), antidepressants (19\%), alcohol (15\%), and carbon monoxide (10\%). The most frequently co-occurring substances causing death across all manners of poisoning deaths were benzodiazepines/opioids (28\%), antidepressants/ benzodiazepines (15\%), alcohol/ opioids (14\%), antidepressants/opioids (14\%), alcohol/ benzodiazepines (11\%), and alcohol/antidepressants (9\%). For suicides overall, the substances that most frequently caused death were over-the-counter or otherwise unclassified drugs (35\%), opioids (27\%), antidepressants (23\%), benzodiazepines (20\%), carbon monoxide (15\%), and alcohol (13\%).

\section{Suicides}

\section{Sex, Race/Ethnicity, Age Group, and Marital Status}

The 18 NVDRS states included in this report collected data for 2014 concerning 14,805 suicide incidents, which included 14,834 deaths (Table 2). Overall, the crude suicide 
rate was 13.9 per 100,000 population. The rate for males was more than three times the rate for females $(21.9$ and 6.3 per 100,000 population, respectively). Non-Hispanic American Indian/Alaska Natives and non-Hispanic whites had the highest rates of suicide (19.1 and 17.0 per 100,000 population, respectively). The highest rates of suicide by age group occurred among persons aged 45-54 years and 55-64 years (20.4 and 18.9 per 100,000 population, respectively). Persons aged $10-14$ years had the lowest rate of suicide among all age groups (2.4 per 100,000 population).

Decedents aged 35-64 years accounted for more than half (51.8\%) of suicides among males. Rates among males were highest for men aged $\geq 85$ years, followed by men aged $75-84$ and 45-54 years (47.2, 35.1, and 30.1 per 100,000 population, respectively). Non-Hispanic American Indian/Alaska Natives (30.7 per 100,000 population) and non-Hispanic whites (26.6 per 100,000 population) had the highest rates of any male subgroups; these rates were approximately three times the rate for males with the lowest rate, Asian/Pacific Islanders (9.2 per 100,000 population). Decedents aged 35-64 years also accounted for the majority (61.5\%) of suicides among females. The rate among females was highest for women aged 45-54 years (11.0 per 100,000). Non-Hispanic American Indian/Alaska Natives (8.0 per 100,000 population) and nonHispanic whites (7.7 per 100,000 population) had the highest suicide rates among females; rates were lowest among nonHispanic black (2.3 per 100,000 population) and Hispanic (2.8 per 100,000 population) females. Of suicide decedents aged $\geq 18$ years, $34.4 \%$ were married, $31.0 \%$ had never been married, and $22.8 \%$ were divorced at the time of death (Table 2 ).

\section{Method and Location of Injury}

Firearms were used in more than half of suicides (51.1\%), followed by hanging/strangulation/suffocation (27.0\%) and poisoning $(15.2 \%)$ (rates: $7.1,3.8$, and 2.1 per 100,000 population, respectively) (Table 3 ). Among males, the most common method used was a firearm $(56.6 \%)$, followed by hanging/strangulation/suffocation (27.0\%) (Table 3). Among females, poisoning was the most common method used (33.4\%), followed by a firearm $(32.6 \%)$. The most common place of suicide was a house or apartment $(75.3 \%)$, followed by a natural area $(4.6 \%)$, a motor vehicle $(4.4 \%)$, a hotel $/$ motel (2.4\%), and a street/highway (2.3\%).

\section{Toxicology Results of Decedent}

Tests for alcohol were conducted for $53.1 \%$ of suicide decedents. Tests for amphetamines, antidepressants, benzodiazepines, cocaine, marijuana, and opiates were conducted for $31.1 \%, 26.0 \%, 33.7 \%, 32.6 \%, 29.0 \%$, and $36.2 \%$, respectively (Table 4 ). Among those with positive results for alcohol $(40.2 \%), 69.4 \%$ had a blood alcohol concentration $(\mathrm{BAC}) \geq 0.08 \mathrm{~g} / \mathrm{dL}$. Opiates (including heroin and prescription pain medications) were identified in $30.0 \%$ of decedents tested for these substances; cocaine and marijuana were identified in $5.7 \%$ and $21.0 \%$ of decedents tested, respectively. Of those tested for antidepressants, $40.8 \%$ had positive results at the time of their death, and $32.6 \%$ of those tested for benzodiazepines had positive results (Table 4).

\section{Precipitating Circumstances}

Precipitating circumstances were known for $90.1 \%$ of suicides. Overall, mental health problems were the most common circumstance; $36.6 \%$ of decedents were described as experiencing a depressed mood at the time of their death, $47.7 \%$ as having a current diagnosed mental health problem, and $28.3 \%$ as receiving mental health treatment (Table 5). Among the 6,375 decedents with a current diagnosed mental health problem, depression/dysthymia $(73.3 \%)$, anxiety disorder (15.9\%), and bipolar disorder (15.0\%) were the most common diagnoses for both males and females (Table 6).

Equivalent percentages of both male $(36.6 \%)$ and female $(36.5 \%)$ decedents were reported to have a depressed mood at the time of their suicide. Greater percentages of females were reported to have a current diagnosed mental health problem (62.3\% of females and $43.2 \%$ of males) and/or to be receiving current mental health treatment (40.8\% of females and $24.5 \%$ of males) (Table 5).

Among 13,368 suicides with known circumstances, 33.7\% of decedents left a suicide note, $31.6 \%$ had a history of suicidal thoughts or plans, $19.8 \%$ had a history of previous suicide attempts, and $24.6 \%$ had disclosed suicidal intent to another person (Table 5). Of those who disclosed intent, the majority of disclosures were to a previous or current intimate partner (38.5\%) or to some other family member (29.7\%). Alcohol or other substance abuse problems were indicated for $17.9 \%$ and $15.9 \%$ of suicide decedents, respectively. A higher percentage of males (18.6\% of males and $15.6 \%$ of females) reportedly had alcohol problems, and a higher percentage of females had another substance problem indicated (19.3\% of females and $14.9 \%$ of males). Other common circumstances were a crisis in the preceding or upcoming 2 weeks $(34.3 \%)$ and intimate partner problems $(28.3 \%)$. Physical health problems (22.0\%), an argument or conflict (15.3\%), job or financial problems (11.1\% and $9.8 \%$, respectively), family relationship problems $(10.0 \%)$, and recent criminal legal problems $(9.1 \%)$ were also reported to have preceded suicides.

Job problems were noted as a precipitating circumstance in a higher percentage of males than females $(12.3 \%$ and $7.3 \%$, respectively), as were financial problems $(10.5 \%$ and $7.7 \%)$ and recent criminal legal problems (10.7\% and $4.1 \%)$. In contrast, 
family (nonintimate partner) relationship problems were a precipitating circumstance in a higher percentage of suicides of females than males (12.5\% and $9.2 \%$, respectively). Although indicated in a small percentage of suicides, males were more often a perpetrator of interpersonal violence in the month before death $(3.1 \%)$ than were females $(<1.0 \%)$ (Table 5$)$.

\section{Homicides}

\section{Sex, Race/Ethnicity, Age Group, and Marital Status}

The 18 NVDRS states included in this report collected data concerning 4,850 homicide incidents, which included 5,100 deaths in 2014 (Table 7). Overall, the crude homicide rate was 4.8 deaths per 100,000 population. The majority of homicide decedents aged $\geq 18$ years $(61.2 \%)$ had never been married, $19.1 \%$ were married, and $12.6 \%$ were divorced at the time of their death (Table 8 ). In more than half (51.3\%) of homicides, the relationship of the victim to the suspect was not known; when the relationship was known, the suspect most often was an acquaintance or friend (28.1\%), a spouse or intimate partner $(22.6 \%)$, or a stranger $(13.6 \%)$.

The homicide rate for males was approximately three times the rate for females (7.5 and 2.1 per 100,000 population, respectively) (Table 7). Non-Hispanic blacks accounted for more than half (55.5\%) of homicides and had the highest rate (17.3 per 100,000 population), followed by non-Hispanic American Indian/Alaska Natives (9.4 per 100,000 population) and Hispanics (4.3 per 100,000 population). Non-Hispanic black males had the highest rate of homicide deaths among males of any race/ethnicity (31.0 per 100,000 population), over 11 times the homicide rate of non-Hispanic white males $(2.8$ per 100,000). Age-specific homicide rates were highest among persons aged 20-24 years (12.2 per 100,000 population), followed by persons aged 25-29 years (10.9 per 100,000 population). The rate for infants aged $<1$ year was over two and a half times the rate for children aged $1-4$ years (6.1 and 2.2 per 100,000 population, respectively). Rates were lowest among persons aged $5-14$ years and $\geq 55$ years. Among males, the majority of homicide decedents $(64.4 \%)$ were aged $20-44$ years; the rate was highest among men aged $20-24$ years (20.6 per 100,000 population). Among females, the homicide rate was highest among infants aged $<1$ year $(5.5$ per 100,000 population). The rate among male infants aged $<1$ year was 6.6 per 100,000 population.

\section{Method and Location of Injury}

Firearms were used in $68.6 \%$ of homicides, followed by sharp instruments $(13.3 \%)$, blunt instruments $(5.4 \%)$, personal weapons (e.g., hands, feet, or fists) (4.7\%), and hanging/ strangulation/suffocation (3.6\%) (Table 9). No other method was used in more than $1 \%$ of homicides. Firearms were the most common method used in homicides of males (73.5\%) and females (52.2\%) (Table 9). Hanging/strangulation/ suffocation was more common among females $(8.7 \%)$ than males $(2.1 \%)$, as was use of blunt instruments $(8.1 \%$ and $4.6 \%$, respectively). A house or apartment was the most common location of homicide $(51.8 \%$ : males and females $45.9 \%$ and $72.0 \%$, respectively), followed by a street or highway (18.6\%: males and females $21.9 \%$ and $7.5 \%$, respectively), a motor vehicle (7.2\%), and a parking lot, a public garage, or public transport (3.7\%) (Table 9).

\section{Precipitating Circumstances}

Precipitating circumstances were identified for $79.4 \%$ of homicides. More than one in three of those homicides was precipitated by another crime (37.4\%) (Table 10); in 56.4\% of those cases the crime was in progress at the time of the incident. The type of crime most frequently precipitating the homicide was assault/homicide (51.9\%), followed by robbery (32.5\%), burglary $(11.6 \%)$, drug trade** $(9.3 \%)$, rape/sexual assault $(2.7 \%)$, arson $(2.1 \%)$, and motor vehicle theft $(1.7 \%)$. Other common precipitating circumstances were an argument or conflict (36.2\%), a physical fight between two people (12.0\%), drug involvement (11.6\%), and a substance abuse problem other than alcohol abuse (10.3\%). In $18.5 \%$ of homicides with known circumstances, intimate partner violence (IPV) was identified as a contributing factor (Table 10).

IPV was a precipitating factor in $47.6 \%$ of homicides among females but only $8.8 \%$ among males. An argument or a conflict was a factor in more homicides among males (37.9\%) than among females (30.9\%). Physical fights precipitated $14.1 \%$ of homicides of males, but only $5.4 \%$ of homicides of females. Likewise, drug involvement more commonly precipitated homicides of males, accounting for $13.8 \%$ of homicides among males and $5.0 \%$ among females. However, a substance abuse problem other than alcohol abuse was implicated as a circumstance present in roughly equal percentages of males and females (10.1\% and $11.0 \%$, respectively). Gang-related homicides were also more common among males $(6.8 \%)$ than females $(2.6 \%)$. Male decedents also used a weapon during the incident in $6.1 \%$ and female decedents in $<1 \%$ of homicides with known circumstances (Table 10).

\footnotetext{
** Drug trade is defined as the buying, selling, or passing of drugs in exchange for goods or money. Drug involvement includes drug use in addition to drug trade.
} 


\section{Legal Intervention Deaths}

\section{Sex, Race/Ethnicity and Age Group}

The 18 NVDRS states included in this report collected data concerning 281 legal intervention death incidents, which included 283 deaths in 2014 (Table 11). The vast majority of legal intervention deaths occurred among males (93.2\%), with the highest rate among men aged 25-29 years (1.2 per 100,000 population), followed by men aged $30-34$ years ( 1.1 per 100,000), 20-24 years (1.0 per 100,000), and 35-44 years $(0.9$ per 100,000$)$. Non-Hispanic white males accounted for the highest percentage of legal intervention deaths (48.5\%), but non-Hispanic black males had the highest rate $(1.0$ per 100,000 population), followed by Hispanic males ( 0.8 per $100,000)$, two to almost three times the rate for non-Hispanic white males (0.4 per 100,000).

\section{Method and Location of Injury}

Firearms were used in almost all legal intervention deaths (94.7\%) (Table 12). Legal intervention deaths occurred most frequently in a house or apartment $(41.7 \%)$, followed by a street or highway $(24.0 \%)$ and a motor vehicle $(8.5 \%)$ (Table 12$)$.

\section{Precipitating Circumstances}

Precipitating circumstances were identified for $99.6 \%$ of legal intervention deaths. Approximately $88.3 \%$ were reportedly precipitated by another crime (Table 13 ); in $65.9 \%$ of those, the crime was in progress at the time of the incident. The type of crime most frequently precipitating the legal intervention death was assault/homicide $(65.5 \%)$, followed by robbery $(9.6 \%)$, motor vehicle theft (6.4\%), burglary (5.6\%), and drug trade $(2.0 \%)$. Substance abuse problems other than alcohol abuse $(25.2 \%)$ and current diagnosed mental health problem (21.6\%) were the most common circumstances related to mental health/substance abuse. Other notable precipitating circumstances were an argument or conflict (13.8\%), being a perpetrator of interpersonal violence within the past month (8.9\%), drug involvement (8.9\%), and family relationship problems $(6.4 \%)$. In $10.3 \%$ of legal intervention deaths with known circumstances, IPV was identified as a contributing factor (Table 13). The decedent reportedly used a weapon in $72.7 \%$ of legal intervention deaths (Table 13). A recent crisis (within the previous or upcoming 2 weeks) was cited in $13.8 \%$ of legal intervention deaths (Table 13).

\section{Unintentional Firearm Deaths}

\section{Sex, Race/Ethnicity, and Age Group}

The 18 NVDRS states included in this report collected data concerning 144 incidents involving 144 unintentional firearm deaths in 2014 (Table 14). A total of 69 (47.9\%) of these unintentional fatal injuries were self-inflicted, and $62(43.1 \%)$ were known to be inflicted by another person; for the remaining 13 (9.0\%), it was unknown who inflicted the injury. Males accounted for $84.7 \%$ of decedents. The majority $(67.4 \%)$ were non-Hispanic whites, followed by non-Hispanic blacks (25.0\%). Persons aged 10-24 years accounted for approximately $40 \%$ of all unintentional firearm deaths (Table 14).

\section{Firearm Type and Location of Injury}

Handguns were involved in $59.7 \%$ of unintentional firearm deaths, rifles in $17.4 \%$, and shotguns in $13.9 \%$. (Table 14). Of all unintentional firearm deaths, $76.4 \%$ occurred in a house or apartment, followed by natural areas (4.9\%) and on the street/ highway (4.2\%) (Table 14).

\section{Context of the Injury and Associated Circumstances}

The context of the injury or associated circumstances were known for $91.7 \%$ of unintentional firearm deaths (Table 15). Overall, the most common context of injury was playing with a gun $(40.9 \%)$, followed by cleaning the gun $(12.9 \%)$ and showing the gun to others $(9.8 \%)$. The most common associated circumstance was unintentionally pulling the trigger $(26.5 \%)$, followed by mistakenly thinking the gun was unloaded (15.2\%) and mistakenly thinking the magazine was disengaged (8.3\%) (Table 15).

\section{Deaths of Undetermined Intent}

\section{Sex, Race/Ethnicity and Age Group}

The 18 NVDRS states included in this report collected data concerning 2,257 deaths in 2014 for which a determination of intent could not be made (crude rate: 2.1 per 100,000 population). Rates were higher among males than among females (2.7 and 1.6 per 100,000 population, respectively), and non-Hispanic whites accounted for $74.1 \%$ of deaths of undetermined intent. Non-Hispanic American Indian/ Alaska Native decedents had the highest rate (3.0 per 100,000 population). Nearly half (46.3\%) of persons for whom the manner of death was undetermined were aged 35-54 years. Rates were highest among adults aged $45-54$ years ( 4.0 per 100,000 population). 


\section{Method of Injury}

The most common method of injury in deaths of undetermined intent was poisoning $(73.8 \%)$. No other method accounted for $>4.0 \%$ overall. Opiates (including heroin and prescription pain medications) were the most frequently tested (75.7\%) and most frequently detected (77.8\% of those tested) substances among this group.

\section{Precipitating Circumstances}

Precipitating circumstances were known in approximately $86.6 \%$ of deaths of undetermined intent. The most common circumstances were physical health problems (18.9\%), a crisis during the preceding or upcoming 2 weeks (16.5\%), and intimate partner problems (8.4\%). Nonalcohol substance abuse problems $(65.7 \%)$ and alcohol problems $(29.1 \%)$ were common, and $37.8 \%$ had a current diagnosed mental health problem. Among those with a current diagnosed mental health problem, depression/dysthymia (59.5\%), anxiety disorder $(23.5 \%)$, and bipolar disorder $(23.1 \%)$ were the most common diagnoses.

\section{Discussion}

Violent deaths occur among persons of all ages, races, and ethnicities. NVDRS data can help identify populations particularly affected by violence. The system not only provides details on specific manners of violent deaths but also identifies cross-cutting risk factors for multiple types of violence. These details can increase knowledge about the circumstances associated with violence and can help public health authorities develop data-informed, effective approaches to violence prevention.

Violence is preventable. CDC has developed technical packages that present a collection of strategies that represent the best available evidence to help communities and states prevent or reduce public health problems like violence (10). Each technical package — child abuse and neglect, youth violence and associated risk factors, IPV, sexual violence, and suicide - identifies strategies and approaches that are representative of different levels of the social ecology intended to affect individual behaviors as well as the relationship, family, school, community, and societal factors that influence risk and protective factors for violence.

As NVDRS data demonstrate, the majority of violent deaths are suicides, making suicide prevention an important priority. CDC's suicide technical package identifies seven primary strategies that have the greatest potential to prevent suicide and to reduce the immediate and long-term consequences of suicidal behavior (11). These strategies include strengthening economic supports, strengthening access and delivery of suicide care, creating protective environments, promoting connectedness, teaching coping and problem-solving skills, identifying and supporting people at risk, and lessening harms and preventing future risk. The specific approaches to advance these strategies, including strengthening household financial security, community engagement, and parenting skills and family relationships, are intended to work in combination and reinforce each other to effectively prevent suicide and also have cross-cutting impacts on other forms of violence (11).

NVDRS data underscore the importance of relationship problems as a risk factor for suicides and homicides and the importance of developing social and emotional skills (e.g., communication, conflict resolution, and empathy) and supportive relationships as protective factors (12). Violence is a leading cause of death among youth, and early intervention is critical to the prevention of youth violence. CDC's youth violence technical package emphasizes the preventive effects of youth skill development programs as well as prevention approaches that address relationships and influence school and community environments (12).

The promotion of safe, stable, nurturing relationships and environments also is a key component of the prevention of child abuse and neglect (13). CDC's child abuse and neglect technical package highlights several strategies that increase the likelihood that children will have safe, stable, nurturing relationships and environments. These strategies include strengthening economic support to families, changing social norms to support parents and positive parenting, providing quality care and education early in life, and enhancing parenting skills to promote healthy child development (14). Five percent of all homicides captured by NVDRS in 2014 (approximately 200 deaths) were related to abuse or neglect by a caretaker (the vast majority of which were among minors), approximately the same percentage as those related to gang violence and exceeding those related to many other homicide circumstances.

NVDRS homicide circumstance data also indicate that approximately half of homicides with female victims are related to IPV. Support for survivors of IPV to increase safety and lessen harms is an important part of prevention (15). The U.S. Preventive Services Task Force recommends screening women of childbearing age for IPV and referring women who screen positive to intervention services (16). Screening and counseling about abuse should be done in a culturally sensitive and supportive way to address concerns about health and safety (17). An intervention with multiple counseling sessions to assess the risk for danger, discuss prevention options, develop a safety plan, and share appropriate community resources has been reported to reduce recurrence of partner violence among pregnant women (18). Other prevention approaches focus on 
empowering bystanders in the prevention of IPV and sexual violence $(15,19)$ and primary prevention through teaching young persons about safe and healthy relationship behaviors in the early dating years $(15,20-22)$. These approaches and others (e.g., parenting skill and family relationship programs and engaging men and boys as allies in prevention) are described in CDC's recently released IPV prevention technical package (15). Evidence exists for the effectiveness of these programs (15). For example, Safe Dates, which focuses on reducing dating violence among adolescents by enhancing awareness about abusive relationships, changing norms, and teaching skills to develop healthy relationships, has been reported to reduce long-term physical and sexual dating violence (20) and other types of youth violence (21). IPV prevention efforts can benefit from a comprehensive approach that also addresses community- and system-level factors by creating protective environments and strengthening economic supports for families (e.g., through organizational and workplace policies) (15).

Substance use also frequently precedes suicidal and interpersonal violent behavior (23). Poisoning is the most common suicide method for women, the third most common suicide method overall, and the leading mechanism of deaths of undetermined intent. Seventy-five percent of all decedents were tested, and approximately $88 \%$ of decedents with a history of substance abuse had one or more substances present in their system at the time of death. Opioids and over-the-counter drugs were the substances that most frequently caused death in isolation; opioids in combination with benzodiazepines or antidepressants were some of the more commonly occurring combinations of substances that caused death. Unintentional opioid overdose has been recognized as an epidemic (24), and these findings mirror concerns regarding the overdose potential of opioids. In response to these concerns, CDC recently issued an opioid prescribing guideline to encourage safer prescribing practices aimed at reducing the likelihood of patient abuse and overdose (25). In addition, taking into account that more decedents with a history of substance abuse than those without such a history have drugs in their system at the time of death, these findings suggest that comprehensive violence prevention efforts can benefit from strategies that address ongoing substance abuse problems.

NVDRS data can be used to define public health priorities, develop and evaluate programs and policies, and conduct research regarding violent deaths at the state level. For example, a recently published article using North Carolina Violent Death Reporting System (NCVDRS) data focused on improving the case ascertainment of pregnancy-associated suicides and homicides (26). By linking NCVDRS data to North Carolina maternal mortality data, $55.6 \%$ more pregnancy-associated (i.e., among women who were pregnant or postpartum) violent deaths were identified, resulting in higher mortality ratios for suicide (2.3 versus 3.3 deaths per 100,000 live births) and homicide (3.9 versus 6.2 deaths per 100,000 live births). Information provided by NCVDRS data indicated that $18 \%$ of suicides were related to postpartum depression and $65.5 \%$ of homicides were related to IPV. That analysis not only highlights the feasibility of linking NCVDRS with maternal mortality surveillance data but also underscores the importance of using multiple data systems to provide better estimates of the magnitude and circumstances related to pregnancy-associated violent deaths in North Carolina, which could guide prevention strategies. Data from state VDRS programs have been used more broadly to develop statewide violence prevention plans. For example, Wisconsin VDRS data were used to develop the statewide suicide prevention strategy (27).

NVDRS data also have been used in states to develop programs for veterans. For example, Colorado VDRS data are incorporated in general and topical reports concerning suicides among veterans. One report examined first responders and found that suicide victims who were first responders were more likely to have been veterans than the general population of suicide victims in their state (28). Findings from their data led to enhancing ManTherapy, an online suicide prevention program for men, to promote resources focused on positive mental and physical health for first responders, active military personnel, veterans, and their families (https://mantherapy.org).

At the national level, NVDRS data are relevant to two national prevention initiatives: the National Strategy for Suicide Prevention and Healthy People 2020 (29,30). The National Strategy for Suicide Prevention is a comprehensive national agenda for suicide prevention (29). Healthy People 2020 includes specific objectives for reducing the number of suicides, homicides, and firearm-related deaths and increasing the number of states that link data on violent deaths from death certificates, law enforcement reports, and coroner/ medical examiner reports at state and local levels (30). Unlike other sources of data, NVDRS allows changing patterns in circumstances and risk profiles to be examined, which can affect how the rates are interpreted, help guide prevention activities, and monitor progress toward objectives.

Updating to an online platform has simplified NVDRS system operations and management, improved timeliness of data entry and reporting, and enhanced flexibility to adapt quickly to changing information needs in violence surveillance (2). Changes to the system have also improved its capacity for future expansion to additional states (2). 


\section{Limitations}

The findings in this report are subject to at least eight limitations. First, NVDRS data are available from a limited number of states and therefore are not nationally representative. Second, the availability, completeness, and timeliness of data are dependent on partnerships among state VDRS and state health departments, vital statistics registrars' offices, coroners/ medical examiners, and law enforcement personnel. Data sharing and communication among partners are particularly challenging when states have independent county coroner systems rather than a centralized coroner/medical examiner system, a large number of law enforcement jurisdictions, or both. NVDRS incident data might be limited or incomplete for areas in which these data-sharing relations are not fully developed. Third, toxicology data are not collected consistently across all states or for all alcohol and drug categories. Toxicology testing is not conducted for all decedents; therefore, the percentage of those with positive results for specific substances might be affected by selective testing patterns in coroner/ medical examiner offices (31). Fourth, abstractors are limited to the data included in the investigative reports they receive. Reports might not fully reflect all information known about an incident, particularly for homicides and legal intervention deaths, when data are less readily available until after a full investigation and adjudication are completed. Fifth, a single death might be classified differently in different documents (e.g., unintentional in a law enforcement report, homicide in a coroner/medical examiner report, and undetermined on the death certificate). NVDRS abstractors reconcile these discrepancies using standard NVDRS case definitions and select a single manner of death on the basis of all source documents; the manner of death assigned must be consistent with the manner of death noted in at least one source document. Sixth, variations in coding might occur depending on the abstractor's level of experience. For this reason, CDC provides abstractor training, and states conduct blinded reabstraction of cases to test consistency and identify training needs. Seventh, medical and mental health information (e.g., type of condition and whether the decedent was currently receiving treatment) are not often captured directly from medical records but from coroner/medical examiner reports and the decedent's family members and friends. Therefore, the completeness and accuracy of this information are limited by the knowledge of the informant. Finally, protective factor data (i.e., characteristics or circumstances that reduce the risk for violent death) are not collected by NVDRS because of the nature of death certificates, coroner/medical examiner reports, and law enforcement reports, which typically contain only circumstances associated with risk factors.

\section{Conclusion}

Public health surveillance is the foundation for public health practice. Surveillance is essential to monitoring the prevalence and incidence of violence-related fatal injuries, defining priorities, and directing programmatic and violence-prevention activities (32). Development and expansion of NVDRS are crucial to public health efforts at the federal, state, and local levels to reduce violence and the personal, familial, and societal consequences and costs. Further efforts are needed to increase the number of states participating in NVDRS to include all 50 states, U.S. territories, and the District of Columbia, with the ultimate goal of full national representation.

\section{Acknowledgments}

Contributors to this report included participating state Violent Death Reporting Systems; participating state agencies, including state health departments, vital registrars' offices, coroners'/medical examiners' offices, crime laboratories, and local and state law enforcement agencies; partner organizations, including the Safe States Alliance, American Public Health Association, International Association of Chiefs of Police, National Association of Medical Examiners, National Association for Public Health Statistics and Information Systems, National Violence Prevention Network, Council of State and Territorial Epidemiologists, and Association of State and Territorial Health Officials; federal agencies, including the U.S. Department of Justice (Bureau of Justice Statistics and the Federal Bureau of Investigation) and the U.S. Department of the Treasury (Bureau of Alcohol, Tobacco, Firearms and Explosives); other stakeholders, researchers, and foundations, including Harvard University School of Public Health and the Joyce Foundation; and the National Institute for Occupational Safety and Health and National Center for Health Statistics, CDC.

\section{References}

1. CDC. Web-based Injury Statistics Query and Reporting System (WISQARS). Atlanta, GA: CDC; 2016. https://www.cdc.gov/injury/ wisqars/index.html

2. Blair JM, Fowler KA, Jack SP, Crosby AE. The National Violent Death Reporting System: overview and future directions. Inj Prev 2016;22:i6-11. https://doi.org/10.1136/injuryprev-2015-041819

3. Institute of Medicine. Reducing the burden of injury: advancing prevention and treatment. Washington DC: National Academies Press; 1999. https://www.nap.edu/read/6321

4. US Census Bureau. QuickFacts: United States. https://www.census.gov/ quickfacts/

5. Krug EG, Mercy JA, Dahlberg LL, Zwi AB. The world report on violence and health. Lancet 2002;360:1083-8. https://doi.org/10.1016/ S0140-6736(02)11133-0

6. World Health Organization. International classification of diseases, 10th revision. Geneva, Switzerland: World Health Organization; 2007. http:// www.who.int/classifications/icd/en/

7. CDC. National Violent Death Reporting System coding manual revised [Online], Atlanta, GA; 2016. https://www.cdc.gov/violenceprevention/ nvdrs/coding_manual.html 
8. Murphy SL, Xu JQ, Kochanek KD; National Center for Health Statistics. Deaths: final data for 2010. National Vital Statistics Reports volume 61, number 4. Atlanta, GA: US Department of Human Services, CDC, National Center for Health Statistics; 2013. https://www.cdc.gov/nchs/ data/nvsr/nvsr61/nvsr61_04.pdf

9. CDC, National Center for Health Statistics. US census populations with bridged race categories. Atlanta, GA: US Department of Human Services, CDC, National Center for Health Statistics; 2015. https://www.cdc. gov/nchs/nvss/bridged_race.htm

10. CDC. Technical packages for violence prevention: using evidence-based strategies in your violence prevention efforts. Atlanta, GA: US Department of Health and Human Services, CDC; 2017. https://www. cdc.gov/violenceprevention/pub/technical-packages.html

11. Stone D, Holland K, Bartholow B, et al. Preventing suicide: a technical package of policy, programs, and practices. Atlanta, GA: US Department of Health and Human Services, CDC; 2017.

12. David-Ferdon C, Vivolo-Kantor AM, Dahlberg LL, et al. A comprehensive technical package for the prevention of youth violence and associated risk behaviors. Atlanta, GA: US Department of Health and Human Services, CDC; 2016.

13. CDC, National Center for Injury Prevention and Control. Essentials for childhood: steps to create safe, stable, nurturing relationships and environments. Atlanta, GA: US Department of Health and Human Services, CDC, National Center for Injury Prevention and Control; 2014. https://www.cdc.gov/violenceprevention/pdf/essentials_for_ childhood_framework.pdf

14. Forston B, Klevens J, Merrick M, et al. Preventing child abuse and neglect: a technical package for policy, norm, and programmatic activities. Atlanta, GA: US Department of Health and Human Services, CDC; 2016.

15. Niolon PH, Kearns M, Dills J, et al. Preventing intimate partner violence across the lifespan: a technical package of programs, policies and practices. Atlanta, GA: US Department of Health and Human Services, CDC; 2017. https://www.cdc.gov/violenceprevention/pdf/ipvtechnicalpackages.pdf

16. U.S. Preventive Services Task Force. Final recommendation statement: intimate partner violence and abuse of elderly and vulnerable adults: screening. Atlanta, GA: U.S. Preventive Services Task Force; 2014. https://www.uspreventiveservicestaskforce.org/Page/Document/ RecommendationStatementFinal/intimate-partner-violence-and-abuseof-elderly-and-vulnerable-adults-screening

17. Institute of Medicine. Clinical preventive services for women: closing the gaps. Washington DC: Institute of Medicine; 2011. http:// nationalacademies.org/hmd/Reports/2011/Clinical-Preventive-Servicesfor-Women-Closing-the-Gaps/Recommendations.aspx

18. Kiely M, El-Mohandes AA, El-Khorazaty MN, Blake SM, Gantz MG. An integrated intervention to reduce intimate partner violence in pregnancy: a randomized controlled trial. Obstet Gynecol 2010;115: 273-83. https://doi.org/10.1097/AOG.0b013e3181cbd482

19. Coker AL, Fisher BS, Bush HM, et al. Evaluation of the green dot bystander intervention to reduce interpersonal violence among college students across three campuses. Violence Against Women 2014;21:1507-27. https://doi. org/10.1177/1077801214545284
20. Foshee VA, Bauman KE, Ennett ST, Linder GF, Benefield T, Suchindran C. Assessing the long-term effects of the Safe Dates program and a booster in preventing and reducing adolescent dating violence victimization and perpetration. Am J Public Health 2004;94:619-24. https://doi.org/10.2105/AJPH.94.4.619

21. Foshee VA, Reyes LM, Agnew-Brune CB, et al. The effects of the evidence-based Safe Dates dating abuse prevention program on other youth violence outcomes. Prev Sci 2014;15:907-16. https://doi. org/10.1007/s11121-014-0472-4

22. Miller E, Tancredi DJ, McCauley HL, et al. Coaching boys into men: a cluster-randomized controlled trial of a dating violence prevention program. J Adolesc Health 2012;51:431-8. https://doi.org/10.1016/j. jadohealth.2012.01.018

23. Poorolajal J, Haghtalab T, Farhadi M, Darvishi N. Substance use disorder and risk of suicidal ideation, suicide attempt and suicide death: a metaanalysis. J Public Health 2016;38:e282-91. https://doi.org/10.1093/ pubmed/fdv148

24. Rudd RA, Aleshire N, Zibbell JE, Gladden RM. Increases in drug and opioid overdose deaths-United States, 2000-2014. MMWR Morb Mortal Wkly Rep 2016;64:1378-82. https://doi.org/10.15585/mmwr. mm6450a3

25. Dowell D, Haegerich TM, Chou R. CDC guideline for prescribing opioids for chronic pain-United States, 2016. MMWR Recomm Rep 2016;65(No. RR-1). https://doi.org/10.15585/mmwr.rr6501e1

26. Austin AE, Vladutiu CJ, Jones-Vessey KA, Norwood TS, Proescholdbell SK, Menard MK. Improved ascertainment of pregnancy-associated suicides and homicides in North Carolina. Am J Prev Med 2016;51(5S3):S234-40. https://doi.org/10.1016/j.amepre.2016.04.023

27. Prevent Suicide Wisconsin Steering Committee. Wisconsin suicide prevention strategy. Madison, WI: Wisconsin Department of Health Services; 2015. https://www.dhs.wisconsin.gov/publications/p00968.pdf

28. Jamison E, Herndon K, Bui AG, Bol K. Suicide among first responders in Colorado, 2004-2014: A summary from the Colorado Violent Death Reporting System. HealthWatch 2015;97:1-4.

29. Office of the Surgeon General; National Alliance for Suicide Prevention. 2012 national strategy for suicide prevention: goals and objectives for action. Washington, DC: US Department of Health and Human Services; 2012. https://www.surgeongeneral.gov/library/reports/ national-strategy-suicide-prevention/full-report.pdf

30. US Department of Health and Human Services. Healthy people 2020. Washington, DC: CDC; 2013. https://www.healthypeople.gov/2020/ topics-objectives

31. Karch D, Crosby A, Simon T; Toxicology testing and results for suicide victims-13 states, 2004. MMWR Morb Mortal Wkly Rep 2006; 55:1245-8.

32. Steenkamp M, Frazier L, Lipskiy N, et al. The National Violent Death Reporting System: an exciting new tool for public health surveillance. Inj Prev 2006;12(Suppl 2):ii3-5 10.1136/ip.2006.012518. https://doi. org/10.1136/ip.2006.012518 
TABLE 1. Number, ${ }^{*}$ percentage, ${ }^{\dagger}$ and rate $^{\S}$ of deaths, by incident type, manner of death, method used and location in which injury occurred - National Violent Death Reporting System, 18 states, " 2014

\begin{tabular}{|c|c|c|}
\hline Characteristic & No. (\%) & Rate \\
\hline \multicolumn{3}{|l|}{ Incident type } \\
\hline Suicide, single & $14,560(65.9)$ & 13.7 \\
\hline Homicide, single & $4,459(20.2)$ & 4.2 \\
\hline Undetermined intent, single & $2,222(10.1)$ & 2.1 \\
\hline Unintentional firearm, single & $144(<1.0)$ & 0.1 \\
\hline Suicide, multiple & $23(<1.0)$ & ** \\
\hline Homicide, multiple & $169(<1.0)$ & ** \\
\hline Undetermined intent, multiple & $12(<1.0)$ & $* *$ \\
\hline Legal intervention, ${ }^{+\dagger}$ single/multiple & $281(1.3)$ & ** \\
\hline Homicide followed by suicide & $222(1.0)$ & ** \\
\hline Other combinations of deaths & $6(<1.0)$ & $* *$ \\
\hline Total & $22,098(100)$ & 20.7 \\
\hline \multicolumn{3}{|l|}{ Manner of death } \\
\hline Suicide or intentional self-harm & $14,834(65.6)$ & 13.9 \\
\hline Homicide & $5,100(22.5)$ & 4.8 \\
\hline Undetermined intent & $2,257(10.0)$ & 2.1 \\
\hline Legal Intervention ${ }^{\dagger \dagger}$ & $283(1.3)$ & 0.3 \\
\hline Unintentional firearm & $144(<1.0)$ & 0.1 \\
\hline Total & $22,618(100)$ & 21.2 \\
\hline \multicolumn{3}{|l|}{ Method } \\
\hline Firearm & $11,554(51.1)$ & 10.8 \\
\hline Hanging/strangulation/suffocation & $4,243(18.8)$ & 4.0 \\
\hline Poisoning & 3,946 (17.4) & 3.7 \\
\hline Sharp instrument & $955(4.2)$ & 0.9 \\
\hline Blunt instrument & $334(1.5)$ & 0.3 \\
\hline Fall & $320(1.4)$ & 0.3 \\
\hline $\begin{array}{l}\text { Motor vehicle (e.g., car, bus, motorcycle, or } \\
\text { other transport vehicle) }\end{array}$ & $269(1.2)$ & 0.3 \\
\hline Personal weapons (e.g., hands, feet, fists) & $255(1.1)$ & 0.2 \\
\hline Drowning & $214(<1.0)$ & 0.2 \\
\hline Fire/burns & $134(<1.0)$ & 0.1 \\
\hline Intentional neglect & $22(<1.0)$ & 0.0 \\
\hline Other (single method) & $75(<1.0)$ & 0.1 \\
\hline Unknown & $297(1.3)$ & 0.3 \\
\hline Total & $22,618(100)$ & 21.2 \\
\hline
\end{tabular}

TABLE 1. (Continued) Number, ${ }^{*}$ percentage, ${ }^{\dagger}$ and rate $^{\S}$ of deaths, by incident type, manner of death, method used and location in which injury occurred - National Violent Death Reporting System, 18 states," 2014

\begin{tabular}{lrr}
\hline Characteristic & No. $(\%)$ & Rate \\
\hline Location & & \\
House, apartment & $15,713(69.5)$ & 14.7 \\
Street/highway & $1,420(6.3)$ & 1.3 \\
Motor vehicle & $1,105(4.9)$ & 1.0 \\
Natural area & $916(4.0)$ & 0.9 \\
Hotel/motel & $509(2.3)$ & 0.5 \\
Parking lot/public garage/public transport & $404(1.8)$ & 0.4 \\
Commercial/retail area & $303(1.3)$ & 0.3 \\
Jail/prison & $258(1.1)$ & 0.2 \\
Park, playground, sports/athletic area & $238(1.1)$ & 0.2 \\
Bar/nightclub & $118(<1.0)$ & 0.1 \\
Supervised residential facility & $98(<1.0)$ & 0.1 \\
Railroad tracks & $94(<1.0)$ & 0.1 \\
Hospital or medical facility & $78(<1.0)$ & 0.1 \\
Farm & $69(<1.0)$ & 0.1 \\
Abandoned house/building/warehouse & $64(<1.0)$ & 0.1 \\
Industrial or construction area & $58(<1.0)$ & 0.1 \\
Preschool/school/college/school bus & $56(<1.0)$ & 0.1 \\
Office building & $38(<1.0)$ & 0.0 \\
Other & $370(1.6)$ & 0.3 \\
Unknown & $709(3.1)$ & 0.7 \\
Total & $22,618(100)$ & 21.2 \\
\hline
\end{tabular}

${ }^{*}$ No. incidents $=22,098$; no. decedents $=22,368$ (98.9\%); no. suspect/ decedents $=250(1.1 \%)$. The Incident type characteristic reports number of incidents; all others report number of deaths.

$\dagger$ Percentages might not total $100 \%$ due to rounding.

$\S$ Per 100,000 population.

I Alaska, Colorado, Georgia, Kentucky, Maryland, Massachusetts, Michigan, North Carolina, New Jersey, New Mexico, Ohio, Oklahoma, Oregon, Rhode Island, South Carolina, Utah, Virginia, and Wisconsin.

** Because the number of decedents varies in incidents involving multiple deaths, numerators cannot be determined to compute rates.

${ }^{+\dagger}$ The term "legal intervention" does not denote the lawfulness or legality of the circumstances surrounding the death. 
TABLE 2. Number, percentage, ${ }^{*}$ and rate ${ }^{\dagger}$ of suicides, by decedent's sex, age group, race/ethnicity, and marital status — National Violent Death Reporting System, 18 states, $^{\S} 2014$

\begin{tabular}{|c|c|c|c|c|c|c|}
\hline \multirow[b]{2}{*}{ Characteristic } & \multicolumn{2}{|c|}{ Male } & \multicolumn{2}{|c|}{ Female } & \multicolumn{2}{|c|}{ Total } \\
\hline & No. (\%) & Rate & No. (\%) & Rate & No. (\%) & Rate \\
\hline \multicolumn{7}{|l|}{ Age group (yrs) } \\
\hline$<10$ & ๆ & ๆ & ๆ & १ & १ & 9 \\
\hline $10-14$ & $117(1.0)$ & 3.3 & $53(1.6)$ & 1.6 & $170(1.1)$ & 2.4 \\
\hline $15-19$ & $518(4.5)$ & 14.3 & $172(5.1)$ & 5.0 & $690(4.7)$ & 9.8 \\
\hline $20-24$ & $966(8.4)$ & 24.7 & $203(6.0)$ & 5.5 & 1,169 (7.9) & 15.3 \\
\hline $25-29$ & $892(7.8)$ & 24.7 & $218(6.4)$ & 6.2 & $1,110(7.5)$ & 15.5 \\
\hline $30-34$ & $925(8.1)$ & 26.3 & $246(7.2)$ & 7.0 & 1,171 (7.9) & 16.6 \\
\hline $35-44$ & 1,797 (15.7) & 26.8 & $626(18.4)$ & 9.2 & $2,423(16.3)$ & 17.9 \\
\hline $45-54$ & $2,178(19.0)$ & 30.1 & $830(24.4)$ & 11.0 & $3,008(20.3)$ & 20.4 \\
\hline $55-64$ & $1,953(17.1)$ & 29.7 & 635 (18.7) & 9.0 & $2,588(17.4)$ & 18.9 \\
\hline $65-74$ & $1,107(9.7)$ & 26.6 & $257(7.6)$ & 5.4 & $1,364(9.2)$ & 15.3 \\
\hline $75-84$ & $671(5.9)$ & 35.1 & $119(3.5)$ & 4.6 & $790(5.3)$ & 17.7 \\
\hline$\geq 85$ & $313(2.7)$ & 47.2 & $36(1.1)$ & 2.7 & $349(2.4)$ & 17.5 \\
\hline Unknown & $1(<1.0)$ & $* *$ & $0(0.0)$ & $* *$ & $1(<1.0)$ & ** \\
\hline Total & $11,438(100)$ & 21.9 & $3,396(100)$ & 6.3 & $14,834(100)$ & 13.9 \\
\hline \multicolumn{7}{|l|}{ Race/ethnicity } \\
\hline White, non-Hispanic & $9,626(84.2)$ & 26.6 & $2,880(84.8)$ & 7.7 & $12,506(84.3)$ & 17.0 \\
\hline Black, non-Hispanic & $767(6.7)$ & 9.8 & $200(5.9)$ & 2.3 & $967(6.5)$ & 5.9 \\
\hline American Indian/Alaska Native, non-Hispanic & $182(1.6)$ & 30.7 & $49(1.4)$ & 8.0 & $231(1.6)$ & 19.1 \\
\hline Asian/Pacific Islander & $199(1.7)$ & 9.2 & $86(2.5)$ & 3.7 & 285 (1.9) & 6.3 \\
\hline Hispanic ${ }^{\dagger \dagger}$ & $555(4.9)$ & 10.0 & $147(4.3)$ & 2.8 & $702(4.7)$ & 6.5 \\
\hline Other & $71(<1.0)$ & $* *$ & $14(<1.0)$ & $* *$ & $85(<1.0)$ & ** \\
\hline Unknown & $38(<1.0)$ & ** & $20(<1.0)$ & ** & $58(<1.0)$ & ** \\
\hline Total & $11,438(100)$ & 21.9 & $3,396(100)$ & 6.3 & $14,834(100)$ & 13.9 \\
\hline \multicolumn{7}{|l|}{ Marital status $\S \S$} \\
\hline Married & $3,819(34.5)$ & ๆๆ & $1,105(34.2)$ & ๆๆ & $4,924(34.4)$ & ๆๆ \\
\hline Never married & $3,669(33.1)$ & ๆๆ & $769(23.8)$ & १९ & $4,438(31.0)$ & ๆๆ \\
\hline Widowed & $608(5.5)$ & ๆๆ & $254(7.9)$ & ๆๆ & $862(6.0)$ & ๆ१ \\
\hline Divorced & $2,317(20.9)$ & ११ & $953(29.5)$ & ११ & $3,270(22.8)$ & ๆ१ \\
\hline Married, but separated & $367(3.3)$ & ๆๆ & $79(2.4)$ & ११ & $446(3.1)$ & ๆๆ \\
\hline Single, not otherwise specified & $171(1.5)$ & १ๆ & $41(1.3)$ & १९ी & $212(1.5)$ & ๆๆ \\
\hline Unknown & $131(1.2)$ & १ๆ & $34(1.1)$ & ११ & $165(1.2)$ & ๆ१ \\
\hline Total & $11,082(100)$ & १ๆ & $3,235(100)$ & १ๆ & $14,317(100)$ & ๆ१ \\
\hline
\end{tabular}

* Percentages might not total $100 \%$ due to rounding.

† Per 100,000 population.

${ }^{\S}$ Alaska, Colorado, Georgia, Kentucky, Maryland, Massachusetts, Michigan, North Carolina, New Jersey, New Mexico, Ohio, Oklahoma, Oregon, Rhode Island, South Carolina, Utah, Virginia, and Wisconsin.

I Suicide is not reported for decedents aged $<10$ years, as per standard in the suicide prevention literature.

** Rates not reported when number of decedents is <20 or when race/ethnicity or age categories are "Other" or "Unknown."

$\dagger^{+}$Includes persons of any race.

$\S \S$ Includes decedents aged $\geq 18$ years only.

ๆๆ Rates cannot be computed for marital status because denominators are unknown. 
TABLE 3. Number, percentage, ${ }^{*}$ and rate ${ }^{\dagger}$ of suicides, by decedent's sex, method used, and location in which injury occurred — National Violent Death Reporting System, 18 states, $\$ 2014$

\begin{tabular}{|c|c|c|c|c|}
\hline \multirow[b]{2}{*}{ Characteristic } & \multirow{2}{*}{$\begin{array}{c}\text { Male } \\
\text { No. (\%) }\end{array}$} & \multirow{2}{*}{$\begin{array}{l}\text { Female } \\
\text { No. (\%) }\end{array}$} & \multicolumn{2}{|c|}{ Total } \\
\hline & & & No. (\%) & Rate \\
\hline \multicolumn{5}{|l|}{ Method } \\
\hline Firearm & $6,469(56.6)$ & $1,106(32.6)$ & $7,575(51.1)$ & 7.1 \\
\hline Hanging/strangulation/suffocation & $3,091(27.0)$ & $907(26.7)$ & $3,998(27.0)$ & 3.8 \\
\hline Poisoning & $1,125(9.8)$ & $1,134(33.4)$ & $2,259(15.2)$ & 2.1 \\
\hline Fall & $206(1.8)$ & $59(1.7)$ & $265(1.8)$ & 0.2 \\
\hline Sharp instrument & $210(1.8)$ & $54(1.6)$ & $264(1.8)$ & 0.2 \\
\hline Motor vehicles (e.g., car, bus, motorcycle, or other transport vehicle) & $146(1.3)$ & $48(1.4)$ & $194(1.3)$ & 0.2 \\
\hline Drowning & $83(<1.0)$ & $49(1.4)$ & $132(<1.0)$ & 0.1 \\
\hline Fire/burns & $39(<1.0)$ & $17(<1.0)$ & $56(<1.0)$ & 0.1 \\
\hline Other (single method) & $23(<1.0)$ & $5(<1.0)$ & $28(<1.0)$ & 0.0 \\
\hline Unknown & $32(<1.0)$ & $12(<1.0)$ & $44(<1.0)$ & 0.0 \\
\hline Total & $11,438(100)$ & $3,396(100)$ & $14,834(100)$ & 13.9 \\
\hline \multicolumn{5}{|l|}{ Location } \\
\hline House, apartment & $8,453(73.9)$ & $2,716(80.0)$ & $11,169(75.3)$ & 10.5 \\
\hline Natural area & $573(5.0)$ & $113(3.3)$ & $686(4.6)$ & 0.6 \\
\hline Motor vehicle & $528(4.6)$ & $129(3.8)$ & $657(4.4)$ & 0.6 \\
\hline Hotel/motel & $248(2.2)$ & $108(3.2)$ & $356(2.4)$ & 0.3 \\
\hline Street/highway & $282(2.5)$ & $52(1.5)$ & $334(2.3)$ & 0.3 \\
\hline Jail/prison & $195(1.7)$ & $22(<1.0)$ & $217(1.5)$ & 0.2 \\
\hline Park, playground, sports/athletic area & $158(1.4)$ & $19(<1.0)$ & $177(1.2)$ & 0.2 \\
\hline Parking lot/public garage/public transport & $140(1.2)$ & $33(<1.0)$ & $173(1.2)$ & 0.2 \\
\hline Commercial/retail area & $96(<1.0)$ & $13(<1.0)$ & $109(<1.0)$ & 0.1 \\
\hline Railroad tracks & $59(<1.0)$ & $18(<1.0)$ & $77(<1.0)$ & 0.1 \\
\hline Farm & $59(<1.0)$ & $2(<1.0)$ & $61(<1.0)$ & 0.1 \\
\hline Hospital or medical facility & $39(<1.0)$ & $17(<1.0)$ & $56(<1.0)$ & 0.1 \\
\hline Supervised residential facility & $40(<1.0)$ & $16(<1.0)$ & $56(<1.0)$ & 0.1 \\
\hline Preschool/school/college/school bus & $40(<1.0)$ & $6(<1.0)$ & $46(<1.0)$ & 0.0 \\
\hline Industrial or construction area & $38(<1.0)$ & $0(0.0)$ & $38(<1.0)$ & 0.0 \\
\hline Office building & $26(<1.0)$ & $4(<1.0)$ & $30(<1.0)$ & 0.0 \\
\hline Other & $223(1.9)$ & $56(1.6)$ & $279(1.9)$ & 0.3 \\
\hline Unknown & $222(1.9)$ & $70(2.1)$ & $292(2.0)$ & 0.3 \\
\hline Total & $11,438(100)$ & $3,396(100)$ & $14,834(100)$ & 13.9 \\
\hline
\end{tabular}

* Percentages might not total $100 \%$ due to rounding.

† Per 100,000 population.

$\S$ Alaska, Colorado, Georgia, Kentucky, Maryland, Massachusetts, Michigan, North Carolina, New Jersey, New Mexico, Ohio, Oklahoma, Oregon, Rhode Island, South Carolina, Utah, Virginia, and Wisconsin. 
TABLE 4. Number* and percentage of suicide decedents who were tested for alcohol and drugs whose results were positive, ${ }^{\dagger}$ by toxicology variable — National Violent Death Reporting System, 18 states, ${ }^{\S} 2014$

\begin{tabular}{|c|c|c|}
\hline & Tested & Positive \\
\hline Toxicology variable & No. (\%) & No. (\%) \\
\hline$B A C^{q}$ & $7,883(53.1)$ & $3,168(40.2)$ \\
\hline Alcohol $<0.08 \mathrm{~g} / \mathrm{dL}$ & & $874(27.6)$ \\
\hline Alcohol $\geq 0.08 \mathrm{~g} / \mathrm{dL}$ & & $2,200(69.4)$ \\
\hline Alcohol positive, level unknown & & $94(3.0)$ \\
\hline Amphetamines & $4,619(31.1)$ & $398(8.6)$ \\
\hline Anticonvulsants & $3,126(21.1)$ & $457(14.6)$ \\
\hline Antidepressants & $3,862(26.0)$ & $1,577(40.8)$ \\
\hline Antipsychotics & $3,072(20.7)$ & $308(10.0)$ \\
\hline Barbiturates & $3,748(25.3)$ & $119(3.2)$ \\
\hline Benzodiazepines & $4,996(33.7)$ & $1,631(32.6)$ \\
\hline Carbon monoxide & $1,805(12.2)$ & 354 (19.6) \\
\hline Cocaine & 4,837 (32.6) & $274(5.7)$ \\
\hline Marijuana & $4,309(29.0)$ & $903(21.0)$ \\
\hline Muscle relaxants & $3,111(21.0)$ & $245(7.9)$ \\
\hline Opiates & $5,368(36.2)$ & $1,611(30.0)$ \\
\hline Other drugs/substances** & 2,654 (17.9) & $2,589(97.6)$ \\
\hline
\end{tabular}

Abbreviation: $\mathrm{BAC}=$ blood alcohol concentration.

* No. of suicide decedents $=14,834$.

† Percentage is of decedents tested for toxicology variable.

$\S$ Alaska, Colorado, Georgia, Kentucky, Maryland, Massachusetts, Michigan, North Carolina, New Jersey, New Mexico, Ohio, Oklahoma, Oregon, Rhode Island, South Carolina, Utah, Virginia, and Wisconsin.

" $\mathrm{BAC}$ of $\geq 0.08 \mathrm{~g} / \mathrm{dL}$ is over the legal limit in all states and is used as the standard for intoxication.

** Other drugs/substances indicated if any results were positive; levels for these drugs/substances are not measured. 
TABLE 5. Number* and percentage ${ }^{\dagger}$ of suicides, by precipitating circumstances and decedent's sex — National Violent Death Reporting System, 18 states, $^{\S} 2014$

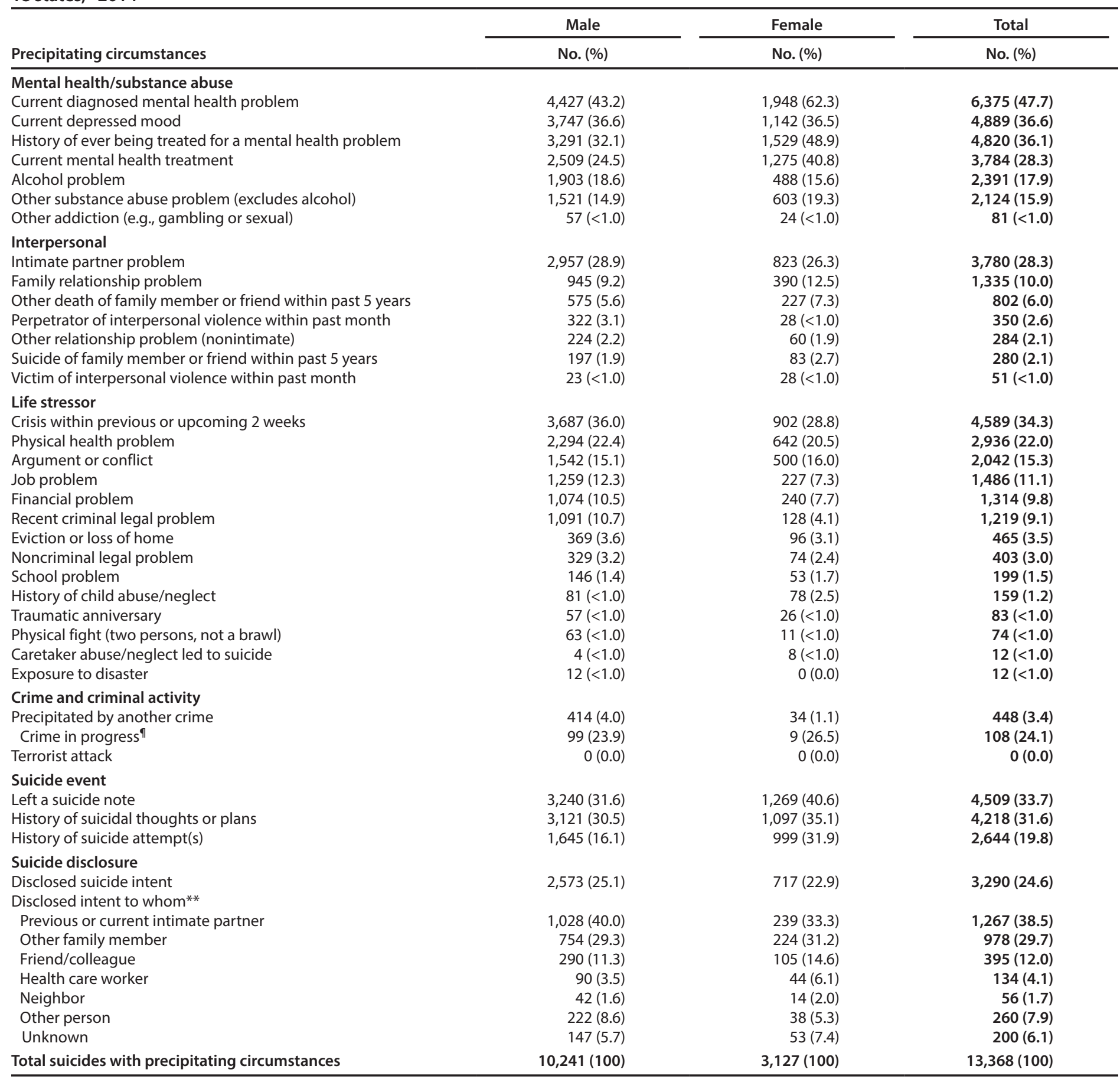

\footnotetext{
* Includes suicides with one or more precipitating circumstances. Circumstances were unknown for 1,466 decedents (1,197 males and 269 females). Numbers do not equal the sums of the columns because more than one circumstance could have been present per decedent.

† Denominator includes only those suicides with one or more precipitating circumstances. The sum of percentages in columns exceeds $100 \%$ because more than one circumstance could have been present per decedent.

$\S$ Alaska, Colorado, Georgia, Kentucky, Maryland, Massachusetts, Michigan, North Carolina, New Jersey, New Mexico, Ohio, Oklahoma, Oregon, Rhode Island, South Carolina, Utah, Virginia, and Wisconsin.

" Denominator includes only those decedents involved in an incident that was precipitated by another crime. Suicide deaths precipitated by this circumstance include situations such as when the suicide victim also committed a homicide or attempted homicide.

** Denominator is decedents who disclosed intent.
} 
TABLE 6. Number* and percentage ${ }^{\dagger}$ of suicide decedents with a current mental health problem, by diagnosis — National Violent Death Reporting System, 18 states, $^{\S} 2014$

\begin{tabular}{|c|c|c|c|}
\hline & Male & Female & Total \\
\hline Mental health problem & No. (\%) & No. (\%) & No. (\%) \\
\hline Depression/dysthymia & $3,207(72.4)$ & $1,469(75.4)$ & $4,676(73.3)$ \\
\hline Anxiety disorder & $652(14.7)$ & $361(18.5)$ & $1,013(15.9)$ \\
\hline Bipolar disorder & $574(13.0)$ & $383(19.7)$ & $957(15.0)$ \\
\hline PTSD & $251(5.7)$ & $55(2.8)$ & $306(4.8)$ \\
\hline Schizophrenia & $231(5.2)$ & $71(3.6)$ & $302(4.7)$ \\
\hline ADD/ADHD & $128(2.9)$ & $31(1.6)$ & $159(2.5)$ \\
\hline OCD & $29(<1.0)$ & $10(<1.0)$ & $39(<1.0)$ \\
\hline Eating disorder & $4(<1.0)$ & $21(1.1)$ & $25(<1.0)$ \\
\hline Other & $227(5.1)$ & $106(5.4)$ & $333(5.2)$ \\
\hline Unknown & $431(9.7)$ & $161(8.3)$ & $592(9.3)$ \\
\hline $\begin{array}{l}\text { Total decedents with a current diagnosed } \\
\text { mental health problem }\end{array}$ & $4,427(100)$ & $1,948(100)$ & $6,375(100)$ \\
\hline
\end{tabular}

Abbreviation: $\mathrm{ADD} / \mathrm{ADHD}=$ attention deficit disorder/attention deficit hyperactivity disorder $\mathrm{OCD}=$ obsessive-compulsive disorder; $\mathrm{PTSD}=$ posttraumatic stress disorder.

* Includes decedents with one or more diagnosed mental health problem. Numbers do not equal the sum of the column because more than one diagnosis could have been present per decedent.

${ }^{\dagger}$ Denominator includes only those decedents with one or more diagnosed mental health problem. Sums of percentages in columns exceed $100 \%$ because decedents could have had more than one diagnosis.

$\S$ Alaska, Colorado, Georgia, Kentucky, Maryland, Massachusetts, Michigan, North Carolina, New Jersey, New Mexico, Ohio, Oklahoma, Oregon, Rhode Island, South Carolina, Utah, Virginia, and Wisconsin.

TABLE 7. Number, percentage, ${ }^{*}$ and rate $^{\dagger}$ of homicides, by decedent's sex, age group, and race/ethnicity — National Violent Death Reporting System, 18 states, $\$ 2014$

\begin{tabular}{|c|c|c|c|c|c|c|}
\hline \multirow[b]{2}{*}{ Characteristic } & \multicolumn{2}{|c|}{ Male } & \multicolumn{2}{|c|}{ Female } & \multicolumn{2}{|c|}{ Total } \\
\hline & No. (\%) & Rate & No. (\%) & Rate & No. (\%) & Rate \\
\hline \multicolumn{7}{|l|}{ Age group (yrs) } \\
\hline$<1$ & $44(1.1)$ & 6.6 & $35(3.0)$ & 5.5 & 79 (1.5) & 6.1 \\
\hline $1-4$ & $67(1.7)$ & 2.5 & $50(4.3)$ & 1.9 & $118(2.3)$ & 2.2 \\
\hline $5-9$ & $21(<1.0)$ & 0.6 & $19(1.6)$ & १ & $40(<1.0)$ & 0.6 \\
\hline $10-14$ & $37(<1.0)$ & 1.0 & $20(1.7)$ & 0.6 & $57(1.1)$ & 0.8 \\
\hline $15-19$ & $311(7.9)$ & 8.6 & $58(5.0)$ & 1.7 & $369(7.2)$ & 5.2 \\
\hline $20-24$ & $805(20.4)$ & 20.6 & $126(10.9)$ & 3.4 & $931(18.3)$ & 12.2 \\
\hline $25-29$ & $660(16.7)$ & 18.2 & $117(10.1)$ & 3.3 & 777 (15.2) & 10.9 \\
\hline $30-34$ & $476(12.1)$ & 13.5 & $127(11.0)$ & 3.6 & $603(11.8)$ & 8.6 \\
\hline $35-44$ & $598(15.2)$ & 8.9 & $199(17.2)$ & 2.9 & 797 (15.6) & 5.9 \\
\hline $45-54$ & 471 (11.9) & 6.5 & 168 (14.6) & 2.2 & 639 (12.5) & 4.3 \\
\hline $55-64$ & $261(6.6)$ & 4.0 & $104(9.0)$ & 1.5 & $365(7.2)$ & 2.7 \\
\hline $65-74$ & $129(3.3)$ & 3.1 & $62(5.4)$ & 1.3 & $191(3.7)$ & 2.1 \\
\hline $75-84$ & $51(1.3)$ & 2.7 & $44(3.8)$ & 1.7 & $95(1.9)$ & 2.1 \\
\hline$\geq 85$ & $10(<1.0)$ & ף & $24(2.1)$ & 1.8 & $34(<1.0)$ & 1.7 \\
\hline Unknown & $4(<1.0)$ & आ & $1(<1.0)$ & १ & $5(<1.0)$ & 9 \\
\hline Total & $3,945(100)$ & 7.5 & $1,154(100)$ & 2.1 & $5,100(100)$ & 4.8 \\
\hline \multicolumn{7}{|l|}{ Race/ethnicity } \\
\hline White, non-Hispanic & $1,006(25.5)$ & 2.8 & $573(49.7)$ & 1.5 & $1,580(31.0)$ & 2.1 \\
\hline Black, non-Hispanic & $2,420(61.3)$ & 31.0 & $412(35.7)$ & 4.8 & $2,832(55.5)$ & 17.3 \\
\hline American Indian/Alaska Native, non-Hispanic & $85(2.2)$ & 14.4 & $28(2.4)$ & 4.6 & $113(2.2)$ & 9.4 \\
\hline Asian/Pacific Islander & $38(<1.0)$ & 1.8 & $21(1.8)$ & 0.9 & $59(1.2)$ & 1.3 \\
\hline Hispanic** & $358(9.1)$ & 6.4 & $107(9.3)$ & 2.0 & $465(9.1)$ & 4.3 \\
\hline Other & $23(<1.0)$ & 9 & $5(<1.0)$ & 9 & $28(<1.0)$ & 9 \\
\hline Unknown & $15(<1.0)$ & १ & $8(<1.0)$ & 9 & $23(<1.0)$ & १ \\
\hline Total & $3,945(100)$ & 7.5 & $1,154(100)$ & 2.1 & $5,100(100)$ & 4.8 \\
\hline
\end{tabular}

* Percentages might not total $100 \%$ due to rounding. Sex was unknown for one decedent.

† Per 100,000 population.

$\S$ Alaska, Colorado, Georgia, Kentucky, Maryland, Massachusetts, Michigan, North Carolina, New Jersey, New Mexico, Ohio, Oklahoma, Oregon, Rhode Island, South Carolina, Utah, Virginia, and Wisconsin.

" Rate is not reported when number of decedents is $<20$ or when age or race/ethnicity is other or unknown.

** Includes persons of any race. 
TABLE 8. Number* and percentage ${ }^{\dagger}$ of homicides, by decedent's marital status and victim-suspect relationship ${ }^{\S}$ - National Violent Death Reporting System, 18 states, " 2014

\begin{tabular}{lr} 
Characteristic & No. (\%) \\
\hline Marital status** & \\
Never married & $2,816(61.2)$ \\
Married & $877(19.1)$ \\
Divorced & $578(12.6)$ \\
Widowed & $148(3.2)$ \\
Single, not otherwise specified & $111(2.4)$ \\
Married, but separated & $68(1.5)$ \\
Total & $4,598(100)$ \\
Relationship & \\
Acquaintance/friend & $698(28.1)$ \\
Spouse/intimate partner (current or former) & $561(22.6)$ \\
Stranger & $337(13.6)$ \\
Other relative & $189(7.6)$ \\
Child & $154(6.2)$ \\
Parent & $139(5.6)$ \\
Other intimate partner involvement & \\
Rival gang member & $58(2.3)$ \\
Victim was law enforcement officer injured in the line of duty & $15(<1.0)$ \\
Other person known to victim & $9(<1.0)$ \\
Victim injured by a law enforcement officer & $326(13.1)$ \\
Total & $0(0.0)$ \\
\hline
\end{tabular}

* No. deaths $=5,100$. Marital status was unknown for 76 decedents. Victimsuspect relationship unknown for 2,614 decedents.

† Percentages might not total $100 \%$ due to rounding.

$\S \mathrm{A}$ victim is a person whose death resulted from a violence-related injury; a suspect is a person believed to have inflicted a fatal injury.

" Alaska, Colorado, Georgia, Kentucky, Maryland, Massachusetts, Michigan, North Carolina, New Jersey, New Mexico, Ohio, Oklahoma, Oregon, Rhode Island, South Carolina, Utah, Virginia, and Wisconsin.

** Includes decedents aged $\geq 18$ years only.

${ }^{+\dagger}$ Death was due to intimate partner-related violence but not between the intimate partners (e.g., child killed by mother's boyfriend). 
TABLE 9. Number, percentage, ${ }^{*}$ and rate $^{\dagger}$ of homicides, by decedent's sex, method used, and location in which injury occurred — National Violent Death Reporting System, 18 states, $\$ 2014$

\begin{tabular}{|c|c|c|c|c|}
\hline \multirow[b]{2}{*}{ Characteristic } & \multirow{2}{*}{$\begin{array}{c}\text { Male } \\
\text { No. (\%) }\end{array}$} & \multirow{2}{*}{$\begin{array}{l}\text { Female } \\
\text { No. (\%) }\end{array}$} & \multicolumn{2}{|c|}{ Total } \\
\hline & & & No. $(\%)$ & Rate \\
\hline \multicolumn{5}{|l|}{ Method } \\
\hline Firearm & $2,899(73.5)$ & $602(52.2)$ & $3,501(68.6)$ & 3.3 \\
\hline Sharp instrument & $482(12.2)$ & $197(17.1)$ & $679(13.3)$ & 0.6 \\
\hline Blunt instrument & $180(4.6)$ & $93(8.1)$ & $273(5.4)$ & 0.3 \\
\hline Personal weapons (e.g., hands, feet, fists) & $165(4.2)$ & $75(6.5)$ & $240(4.7)$ & 0.2 \\
\hline Hanging/strangulation/suffocation & $83(2.1)$ & $100(8.7)$ & $184(3.6)$ & 0.2 \\
\hline Motor vehicle (e.g., car, bus, motorcycle, or other transport vehicle) & $21(<1.0)$ & $14(1.2)$ & $35(<1.0)$ & 0.0 \\
\hline Fire/burns & $15(<1.0)$ & $11(<1.0)$ & $26(<1.0)$ & 0.0 \\
\hline Poisoning & $9(<1.0)$ & $12(1.0)$ & $21(<1.0)$ & 0.0 \\
\hline Intentional neglect & $4(<1.0)$ & $11(<1.0)$ & $15(<1.0)$ & ๆ \\
\hline Fall & $6(<1.0)$ & $5(<1.0)$ & $11(<1.0)$ & १ \\
\hline Drowning & $3(<1.0)$ & $3(<1.0)$ & $6(<1.0)$ & १ \\
\hline Other (single method) & $19(<1.0)$ & $6(<1.0)$ & $25(<1.0)$ & 0.0 \\
\hline Unknown & $59(1.5)$ & $25(2.2)$ & $84(1.6)$ & 0.1 \\
\hline Total & $3,945(100)$ & $1,154(100)$ & $5,100(100)$ & 4.8 \\
\hline \multicolumn{5}{|l|}{ Location } \\
\hline House, apartment & $1,812(45.9)$ & $831(72.0)$ & $2,644(51.8)$ & 2.5 \\
\hline Street/highway & $864(21.9)$ & $86(7.5)$ & 950 (18.6) & 0.9 \\
\hline Motor vehicle & $309(7.8)$ & $58(5.0)$ & $367(7.2)$ & 0.3 \\
\hline Parking lot/public garage/public transport & $169(4.3)$ & $20(1.7)$ & $189(3.7)$ & 0.2 \\
\hline Commercial/retail area & $143(3.6)$ & $18(1.6)$ & $161(3.2)$ & 0.2 \\
\hline Bar/nightclub & $105(2.7)$ & $7(<1.0)$ & $112(2.2)$ & 0.1 \\
\hline Natural area & $83(2.1)$ & $26(2.3)$ & $109(2.1)$ & 0.1 \\
\hline Hotel/motel & $48(1.2)$ & $26(2.3)$ & $74(1.5)$ & 0.1 \\
\hline Park, playground, sports/athletic area & $37(<1.0)$ & $7(<1.0)$ & $44(<1.0)$ & 0.0 \\
\hline Jail/prison & $32(<1.0)$ & $0(0.0)$ & $32(<1.0)$ & 0.0 \\
\hline Abandoned house/building/warehouse & $25(<1.0)$ & $6(<1.0)$ & $31(<1.0)$ & 0.0 \\
\hline Industrial or construction area & $15(<1.0)$ & $3(<1.0)$ & $18(<1.0)$ & १ \\
\hline Supervised residential facility & $9(<1.0)$ & $6(<1.0)$ & $15(<1.0)$ & १ \\
\hline Hospital or medical facility & $7(<1.0)$ & $2(<1.0)$ & $9(<1.0)$ & १ \\
\hline Preschool/school/college/school bus & $6(<1.0)$ & $1(<1.0)$ & $7(<1.0)$ & १ \\
\hline Farm & $4(<1.0)$ & $0(0.0)$ & $4(<1.0)$ & १ \\
\hline Railroad tracks & $3(<1.0)$ & $0(0.0)$ & $3(<1.0)$ & १ \\
\hline Office building & $2(<1.0)$ & $0(0.0)$ & $2(<1.0)$ & १ \\
\hline Other & $55(1.4)$ & $8(<1.0)$ & $63(1.2)$ & 0.1 \\
\hline Unknown & $217(5.5)$ & $49(4.2)$ & $266(5.2)$ & 0.2 \\
\hline Total & $3,945(100)$ & $1,154(100)$ & $5,100(100)$ & 4.8 \\
\hline
\end{tabular}

* Percentages might not total $100 \%$ due to rounding. Sex was unknown for one decedent.

+ Per 100,000 population.

$\S$ Alaska, Colorado, Georgia, Kentucky, Maryland, Massachusetts, Michigan, North Carolina, New Jersey, New Mexico, Ohio, Oklahoma, Oregon, Rhode Island, South Carolina, Utah, Virginia, and Wisconsin.

I Rate is not reported when number of decedents is $<20$. 
TABLE 10. Number* and percentage ${ }^{\dagger}$ of homicides, by precipitating circumstances and decedent's sex — National Violent Death Reporting System, 18 states, ${ }^{\S} 2014$

\begin{tabular}{|c|c|c|c|}
\hline & Male & Female & Total \\
\hline Precipitating circumstances & No. (\%) & No. (\%) & No. (\%) \\
\hline \multicolumn{4}{|l|}{ Mental health/substance abuse } \\
\hline Other substance abuse problem (excludes alcohol) & $306(10.1)$ & $111(11.0)$ & $417(10.3)$ \\
\hline Alcohol problem & $134(4.4)$ & $41(4.1)$ & $175(4.3)$ \\
\hline Current diagnosed mental health problem & $106(3.5)$ & $50(5.0)$ & $156(3.9)$ \\
\hline History of ever being treated for a mental health problem & $62(2.0)$ & $33(3.3)$ & $95(2.3)$ \\
\hline Current mental health treatment & $47(1.5)$ & $25(2.5)$ & $72(1.8)$ \\
\hline Current depressed mood & $9(<1.0)$ & $9(<1.0)$ & $18(<1.0)$ \\
\hline Other addiction (e.g., gambling or sexual) & $3(<1.0)$ & $2(<1.0)$ & $5(<1.0)$ \\
\hline \multicolumn{4}{|l|}{ Interpersonal } \\
\hline Intimate partner violence-related & $269(8.8)$ & $480(47.6)$ & 749 (18.5) \\
\hline Family relationship problem & $183(6.0)$ & $83(8.2)$ & $266(6.6)$ \\
\hline Other relationship problem (nonintimate) & $178(5.9)$ & $35(3.5)$ & $213(5.3)$ \\
\hline Jealousy (lovers' triangle) & $58(1.9)$ & $48(4.8)$ & $106(2.6)$ \\
\hline Victim of interpersonal violence within past month & $27(<1.0)$ & $44(4.4)$ & $71(1.8)$ \\
\hline Perpetrator of interpersonal violence within past month & $37(1.2)$ & $6(<1.0)$ & $43(1.1)$ \\
\hline \multicolumn{4}{|l|}{ Life stressor } \\
\hline Argument or conflict & $1,152(37.9)$ & $312(30.9)$ & $1,464(36.2)$ \\
\hline Physical fight (two persons, not a brawl) & $430(14.1)$ & $54(5.4)$ & $484(12.0)$ \\
\hline Crisis within previous or upcoming 2 weeks & $220(7.2)$ & $98(9.7)$ & $318(7.9)$ \\
\hline History of child abuse/neglect & $25(<1.0)$ & $21(2.1)$ & $46(1.1)$ \\
\hline \multicolumn{4}{|l|}{ Crime and criminal activity } \\
\hline Precipitated by another crime & $1,228(40.4)$ & $285(28.2)$ & $1,513(37.4)$ \\
\hline Crime in progress & $711(57.9)$ & $142(49.8)$ & $853(56.4)$ \\
\hline Drug involvement & $418(13.8)$ & $50(5.0)$ & $468(11.6)$ \\
\hline Gang-related & $207(6.8)$ & $26(2.6)$ & $233(5.8)$ \\
\hline Terrorist attack & $0(0.0)$ & $0(0.0)$ & $0(0.0)$ \\
\hline \multicolumn{4}{|l|}{ Homicide event } \\
\hline Caretaker abuse/neglect led to death & $104(3.4)$ & $101(10.0)$ & $205(5.1)$ \\
\hline Victim used a weapon & $184(6.1)$ & $7(<1.0)$ & $191(4.7)$ \\
\hline Drive-by shooting & $115(3.8)$ & $12(1.2)$ & $127(3.1)$ \\
\hline Walk by assault & $105(3.5)$ & $11(1.1)$ & $116(2.9)$ \\
\hline Justifiable self defense & $105(3.5)$ & $3(<1.0)$ & $108(2.7)$ \\
\hline Brawl & $94(3.1)$ & $7(<1.0)$ & $101(2.5)$ \\
\hline Mentally ill suspect & $60(2.0)$ & $41(4.1)$ & $101(2.5)$ \\
\hline Random violence & $59(1.9)$ & $20(2.0)$ & $79(2.0)$ \\
\hline Victim was a bystander & $39(1.3)$ & $28(2.8)$ & $67(1.7)$ \\
\hline Victim was an intervener assisting a crime victim & $19(<1.0)$ & $3(<1.0)$ & $22(<1.0)$ \\
\hline Prostitution & $12(<1.0)$ & $7(<1.0)$ & $19(<1.0)$ \\
\hline Victim was a police officer on duty & $11(<1.0)$ & $0(0.0)$ & $11(<1.0)$ \\
\hline Stalking & $3(<1.0)$ & $6(<1.0)$ & $9(<1.0)$ \\
\hline Mercy killing & $0(0.0)$ & $6(<1.0)$ & $6(<1.0)$ \\
\hline Hate crime & $2(<1.0)$ & $1(<1.0)$ & $3(<1.0)$ \\
\hline Total homicides with precipitating circumstances & $3,040(100)$ & $1,009(100)$ & $4,049(100)$ \\
\hline
\end{tabular}

* Includes homicides with one or more precipitating circumstances. Total numbers do not equal the sums of the columns because more than one circumstance could have been present per decedent. Circumstances were unknown for 1,051 decedents ( 905 males and 145 females; sex was unknown for one decedent).

† Denominator includes only those homicides with one or more precipitating circumstances. The sum of percentages in columns exceeds $100 \%$ because more than one circumstance could have been present per decedent.

$\S$ Alaska, Colorado, Georgia, Kentucky, Maryland, Massachusetts, Michigan, North Carolina, New Jersey, New Mexico, Ohio, Oklahoma, Oregon, Rhode Island, South Carolina, Utah, Virginia, and Wisconsin.

" Denominator includes only those decedents involved in an incident that was precipitated by another crime. 
TABLE 11. Number, percentage, ${ }^{*}$ and rate $^{\dagger}$ of legal intervention ${ }^{\S}$ deaths, by decedent's sex, age group, and race/ethnicity — National Violent Death Reporting System, 18 states, " 2014

\begin{tabular}{|c|c|c|c|c|c|c|}
\hline \multirow[b]{2}{*}{ Characteristic } & \multicolumn{2}{|c|}{ Male } & \multicolumn{2}{|c|}{ Female } & \multicolumn{2}{|c|}{ Total } \\
\hline & No. (\%) & Rate & No. (\%) & Rate & No. (\%) & Rate \\
\hline \multicolumn{7}{|l|}{ Age group (yrs) } \\
\hline$<1$ & $0(0.0)$ & $* *$ & $0(0.0)$ & $* *$ & $0(0.0)$ & $* *$ \\
\hline $1-4$ & $0(0.0)$ & $* *$ & $0(0.0)$ & $* *$ & $0(0.0)$ & $* *$ \\
\hline $5-9$ & $0(0.0)$ & $* *$ & $0(0.0)$ & $* *$ & $0(0.0)$ & $* *$ \\
\hline $10-14$ & $1(<1.0)$ & $* *$ & $0(0.0)$ & $* *$ & $1(<1.0)$ & $* *$ \\
\hline $15-19$ & $12(4.5)$ & $* *$ & $4(21.1)$ & $* *$ & $16(5.7)$ & $* *$ \\
\hline $20-24$ & 39 (14.8) & 1.0 & $0(0.0)$ & ** & $39(13.8)$ & 0.5 \\
\hline $25-29$ & $45(17.0)$ & 1.2 & $3(15.8)$ & $* *$ & $48(17.0)$ & 0.7 \\
\hline $30-34$ & $38(14.4)$ & 1.1 & $1(5.3)$ & ** & 39 (13.8) & 0.6 \\
\hline $35-44$ & $59(22.3)$ & 0.9 & $7(36.8)$ & $* *$ & $66(23.3)$ & 0.5 \\
\hline $45-54$ & $41(15.5)$ & 0.6 & $2(10.5)$ & ** & $43(15.2)$ & 0.3 \\
\hline $55-64$ & $18(6.8)$ & $* *$ & $1(5.3)$ & $* *$ & $19(6.7)$ & $* *$ \\
\hline $65-74$ & $10(3.8)$ & $* *$ & $1(5.3)$ & ** & $11(3.9)$ & $* *$ \\
\hline $75-84$ & $1(<1.0)$ & ** & $0(0.0)$ & ** & $1(<1.0)$ & $* *$ \\
\hline$\geq 85$ & $0(0.0)$ & $* *$ & $0(0.0)$ & $* *$ & $0(0.0)$ & $* *$ \\
\hline Unknown & $0(0.0)$ & $* *$ & $0(0.0)$ & $* *$ & $0(0.0)$ & $* *$ \\
\hline Total & $264(100)$ & 0.5 & $19(100)$ & $* *$ & $283(100)$ & 0.3 \\
\hline \multicolumn{7}{|l|}{ Race/ethnicity } \\
\hline White, non-Hispanic & $128(48.5)$ & 0.4 & $12(63.2)$ & ** & $140(49.5)$ & 0.2 \\
\hline Black, non-Hispanic & 79 (29.9) & 1.0 & $4(21.1)$ & $* *$ & $83(29.3)$ & 0.5 \\
\hline American Indian/Alaska Native, non-Hispanic & $9(3.4)$ & ** & $0(0.0)$ & $* *$ & $9(3.2)$ & $* *$ \\
\hline Asian/Pacific Islander & $2(<1.0)$ & $* *$ & $1(5.3)$ & ** & $3(1.1)$ & $* *$ \\
\hline Hispanic $^{\dagger \dagger}$ & $45(17.0)$ & 0.8 & $2(10.5)$ & ** & $47(16.6)$ & 0.4 \\
\hline Other & $1(<1.0)$ & $* *$ & $0(0.0)$ & $* *$ & $1(<1.0)$ & $* *$ \\
\hline Unknown & $0(0.0)$ & $* *$ & $0(0.0)$ & $* *$ & $0(0.0)$ & $* *$ \\
\hline Total & $264(100)$ & 0.5 & $19(100)$ & $* *$ & $283(100)$ & 0.3 \\
\hline
\end{tabular}

* Percentages might not total $100 \%$ due to rounding.

+ Per 100,000 population.

$\S$ The term "legal intervention" does not denote the lawfulness or legality of the circumstances surrounding the death.

I Alaska, Colorado, Georgia, Kentucky, Maryland, Massachusetts, Michigan, North Carolina, New Jersey, New Mexico, Ohio, Oklahoma, Oregon, Rhode Island, South Carolina, Utah, Virginia, and Wisconsin.

** Rate is not reported when number of decedents is $<20$ or when race/ethnicity or age categories are "Other" or "Unknown."

${ }^{+\dagger}$ Includes persons of any race. 
TABLE 12. Number, percentage, ${ }^{*}$ and rate ${ }^{\dagger}$ of legal intervention ${ }^{\S}$ deaths, by decedent's sex, method used, and location in which injury occurred — National Violent Death Reporting System, 18 states, " 2014

\begin{tabular}{|c|c|c|c|c|}
\hline \multirow[b]{2}{*}{ Characteristic } & \multirow{2}{*}{$\begin{array}{c}\text { Male } \\
\text { No. (\%) }\end{array}$} & \multirow{2}{*}{$\begin{array}{l}\text { Female } \\
\text { No. (\%) }\end{array}$} & \multicolumn{2}{|c|}{ Total } \\
\hline & & & No. (\%) & Rate \\
\hline \multicolumn{5}{|l|}{ Method } \\
\hline Firearm & $251(95.1)$ & $17(89.5)$ & $268(94.7)$ & 0.3 \\
\hline Motor vehicle (e.g., car, bus, motorcycle, or other transport vehicle) & $3(1.1)$ & $0(0.0)$ & $3(1.1)$ & ** \\
\hline Sharp instrument & $2(<1.0)$ & $0(0.0)$ & $2(<1.0)$ & ** \\
\hline Drowning & $1(<1.0)$ & $0(0.0)$ & $1(<1.0)$ & ** \\
\hline Fall & $1(<1.0)$ & $0(0.0)$ & $1(<1.0)$ & ** \\
\hline Hanging/strangulation/suffocation & $1(<1.0)$ & $0(0.0)$ & $1(<1.0)$ & ** \\
\hline Personal weapons (e.g., hands, feet, fists) & $1(<1.0)$ & $0(0.0)$ & $1(<1.0)$ & ** \\
\hline Poisoning & $1(<1.0)$ & $0(0.0)$ & $1(<1.0)$ & ** \\
\hline Blunt instrument & $0(0.0)$ & $0(0.0)$ & $0(0.0)$ & ** \\
\hline Fire/burns & $0(0.0)$ & $0(0.0)$ & $0(0.0)$ & $* *$ \\
\hline Intentional neglect & $0(0.0)$ & $0(0.0)$ & $0(0.0)$ & ** \\
\hline Other (single method) & $3(1.1)$ & $1(5.3)$ & $4(1.4)$ & ** \\
\hline Unknown & $0(0.0)$ & $1(5.3)$ & $1(<1.0)$ & ** \\
\hline Total & $264(100)$ & $19(100)$ & $283(100)$ & 0.3 \\
\hline \multicolumn{5}{|l|}{ Location } \\
\hline House, apartment & $107(40.5)$ & $11(57.9)$ & $118(41.7)$ & 0.1 \\
\hline Street/highway & $65(24.6)$ & $3(15.8)$ & $68(24.0)$ & 0.1 \\
\hline Motor vehicle & $21(8.0)$ & $3(15.8)$ & $24(8.5)$ & 0.0 \\
\hline Parking lot/public garage/public transport & $19(7.2)$ & $0(0.0)$ & $19(6.7)$ & $* *$ \\
\hline Commercial/retail area & $17(6.4)$ & $0(0.0)$ & $17(6.0)$ & ** \\
\hline Natural area & $10(3.8)$ & $0(0.0)$ & $10(3.5)$ & ** \\
\hline Office building & $3(1.1)$ & $0(0.0)$ & $3(1.1)$ & ** \\
\hline Park, playground, sports/athletic area & $3(1.1)$ & $0(0.0)$ & $3(1.1)$ & *** \\
\hline Farm & $2(<1.0)$ & $0(0.0)$ & $2(<1.0)$ & $* *$ \\
\hline Industrial or construction area & $1(<1.0)$ & $1(5.3)$ & $2(<1.0)$ & ** \\
\hline Jail/prison & $2(<1.0)$ & $0(0.0)$ & $2(<1.0)$ & ** \\
\hline Supervised residential facility & $2(<1.0)$ & $0(0.0)$ & $2(<1.0)$ & ** \\
\hline Bar/nightclub & $1(<1.0)$ & $0(0.0)$ & $1(<1.0)$ & ** \\
\hline Hotel/motel & $1(<1.0)$ & $0(0.0)$ & $1(<1.0)$ & ** \\
\hline Abandoned house/building/warehouse & $0(0.0)$ & $0(0.0)$ & $0(0.0)$ & ** \\
\hline Hospital or medical facility & $0(0.0)$ & $0(0.0)$ & $0(0.0)$ & ** \\
\hline Preschool/school/college/school bus & $0(0.0)$ & $0(0.0)$ & $0(0.0)$ & ** \\
\hline Railroad tracks & $0(0.0)$ & $0(0.0)$ & $0(0.0)$ & ** \\
\hline Other & $9(3.4)$ & $1(5.3)$ & $10(3.5)$ & ** \\
\hline Unknown & $1(<1.0)$ & $0(0.0)$ & $1(<1.0)$ & ** \\
\hline Total & $264(100)$ & $19(100)$ & $283(100)$ & 0.3 \\
\hline
\end{tabular}

* Percentages might not total $100 \%$ due to rounding.

+ Per 100,000 population.

$\S$ The term "legal intervention" does not denote the lawfulness or legality of the circumstances surrounding the death.

" Alaska, Colorado, Georgia, Kentucky, Maryland, Massachusetts, Michigan, North Carolina, New Jersey, New Mexico, Ohio, Oklahoma, Oregon, Rhode Island, South Carolina, Utah, Virginia, and Wisconsin.

** Rate is not reported when number of decedents is $<20$. 
TABLE 13. Number* and percentage ${ }^{\dagger}$ of legal intervention ${ }^{\S}$ deaths, by precipitating circumstances and decedent's sex - National Violent Death Reporting System, 18 states, ${ }^{\text {ๆ }} 2014$

\begin{tabular}{|c|c|c|c|}
\hline & Male & Female & Total \\
\hline Precipitating circumstances & No. (\%) & No. $(\%)$ & No. (\%) \\
\hline \multicolumn{4}{|l|}{ Mental health/substance abuse } \\
\hline Other substance abuse problem (excludes alcohol) & $67(25.5)$ & $4(21.1)$ & $71(25.2)$ \\
\hline Current diagnosed mental health problem & $52(19.8)$ & $9(47.4)$ & $61(21.6)$ \\
\hline History of ever being treated for a mental health problem & $47(17.9)$ & $6(31.6)$ & $53(18.8)$ \\
\hline Current mental health treatment & $31(11.8)$ & $5(26.3)$ & $36(12.8)$ \\
\hline Alcohol problem & $25(9.5)$ & $4(21.1)$ & $29(10.3)$ \\
\hline Current depressed mood & $19(7.2)$ & $2(10.5)$ & $21(7.4)$ \\
\hline Other addiction (e.g., gambling, sexual) & $0(0.0)$ & $0(0.0)$ & $0(0.0)$ \\
\hline \multicolumn{4}{|l|}{ Interpersonal } \\
\hline Intimate partner violence-related & $27(10.3)$ & $2(10.5)$ & $29(10.3)$ \\
\hline Perpetrator of interpersonal violence within past month & $24(9.1)$ & $1(5.3)$ & $25(8.9)$ \\
\hline Family relationship problem & $15(5.7)$ & $3(15.8)$ & $18(6.4)$ \\
\hline Other relationship problem (nonintimate) & $3(1.1)$ & $3(15.8)$ & $6(2.1)$ \\
\hline Jealousy (lovers' triangle) & $3(1.1)$ & $0(0.0)$ & $3(1.1)$ \\
\hline Victim of interpersonal violence within past month & $0(0.0)$ & $0(0.0)$ & $0(0.0)$ \\
\hline \multicolumn{4}{|l|}{ Life stressor } \\
\hline Crisis within previous or upcoming two weeks & $33(12.5)$ & $6(31.6)$ & 39 (13.8) \\
\hline Argument or conflict & $35(13.3)$ & $4(21.1)$ & $39(13.8)$ \\
\hline Physical fight (two persons, not a brawl) & $14(5.3)$ & $2(10.5)$ & $16(5.7)$ \\
\hline History of child abuse/neglect & $3(1.1)$ & $2(10.5)$ & $5(1.8)$ \\
\hline \multicolumn{4}{|l|}{ Crime and criminal activity } \\
\hline Precipitated by another crime & $236(89.7)$ & $13(68.4)$ & $249(88.3)$ \\
\hline Crime in progress** & $158(66.9)$ & $6(46.2)$ & $164(65.9)$ \\
\hline Drug involvement & $23(8.7)$ & $2(10.5)$ & $25(8.9)$ \\
\hline Gang-related & $2(<1.0)$ & $0(0.0)$ & $2(<1.0)$ \\
\hline Terrorist attack & $0(0.0)$ & $0(0.0)$ & $0(0.0)$ \\
\hline \multicolumn{4}{|l|}{ Legal intervention event } \\
\hline Victim used a weapon & $195(74.1)$ & $10(52.6)$ & $205(72.7)$ \\
\hline Brawl & $4(1.5)$ & $0(0.0)$ & $4(1.4)$ \\
\hline Victim was a bystander & $1(<1.0)$ & $1(5.3)$ & $2(<1.0)$ \\
\hline Mentally ill suspect & $1(<1.0)$ & $0(0.0)$ & $1(<1.0)$ \\
\hline Prostitution & $1(<1.0)$ & $0(0.0)$ & $1(<1.0)$ \\
\hline Victim was a police officer on duty & $0(0.0)$ & $0(0.0)$ & $0(0.0)$ \\
\hline Victim was an intervener assisting a crime victim & $0(0.0)$ & $0(0.0)$ & $0(0.0)$ \\
\hline Random violence & $0(0.0)$ & $0(0.0)$ & $0(0.0)$ \\
\hline Stalking & $0(0.0)$ & $0(0.0)$ & $0(0.0)$ \\
\hline Total legal intervention deaths with precipitating circumstances & $263(100)$ & $19(100)$ & $282(100)$ \\
\hline
\end{tabular}

* Includes deaths with one or more precipitating circumstances. Total numbers do not equal the sums of the columns because more than one circumstance could have been present per decedent. Circumstances were unknown for one male decedent.

t Denominator includes only those deaths with one or more precipitating circumstances. The sum of percentages in columns exceed $100 \%$ because more than one circumstance could have been present per decedent.

$\S$ The term "legal intervention" does not denote the lawfulness or legality of the circumstances surrounding the death.

I Alaska, Colorado, Georgia, Kentucky, Maryland, Massachusetts, Michigan, North Carolina, New Jersey, New Mexico, Ohio, Oklahoma, Oregon, Rhode Island, South Carolina, Utah, Virginia, and Wisconsin.

** Denominator includes only those decedents involved in an incident that was precipitated by another crime. 
TABLE 15. Number* and percentage ${ }^{\dagger}$ of unintentional firearm deaths, by context and circumstances of injury - National Violent Death Reporting System, 18 states, ${ }^{\S} 2014$

Characteristic No. (\%)

Context of injury

Playing with gun

54 (40.9)

Cleaning gun

$17(12.9)$

Showing gun to others

$13(9.8)$

Hunting

$11(8.3)$

Loading/unloading gun

$9(6.8)$

Target shooting

$4(3.0)$

Celebratory firing

xt of injury

$33(25.0)$

Circumstances of injury

Unintentionally pulled trigger

$35(26.5)$

Thought gun was unloaded

$20(15.2)$

Thought unloaded, magazine disengaged

$11(8.3)$

Gun fired due to defect or malfunction

$5(3.8)$

Gun was mistaken for a toy

Thought gun safety was engaged

$2(1.5)$

Bullet ricocheted

Gun fired while holstering

Gun was dropped

$1(<1.0)$

Gun fired while handling safety lock

$1(<1.0)$

Other mechanism of injury

$1(<1.0)$

Total unintentional firearm deaths with precipitating circumstances

* Includes 132 deaths with one or more circumstances known. Circumstances were unknown for 12 deaths.

† Percentages might exceed $100 \%$ because one or more circumstances could have been known per death.

$\S$ Alaska, Colorado, Georgia, Kentucky, Maryland, Massachusetts, Michigan, North Carolina, New Jersey, New Mexico, Ohio, Oklahoma, Oregon, Rhode Island, South Carolina, Utah, Virginia, and Wisconsin. 


The Morbidity and Mortality Weekly Report (MMWR) Series is prepared by the Centers for Disease Control and Prevention (CDC) and is available free of charge in electronic format. To receive an electronic copy each week, visit $M M W R$ 's free subscription page at https://www.cdc.gov/mmwr $/ \mathrm{mmwrsubscribe.html}$. Paper copy subscriptions are available through the Superintendent of Documents, U.S. Government Printing Office, Washington, DC 20402; telephone 202-512-1800

Readers who have difficulty accessing this PDF file may access the HTML file at https://www.cdc.gov/mmwr/volumes/67/ss/ss6702a1.htm?s_ cid=ss6702a1_w. Address all inquiries about the $M M W R$ Series, including material to be considered for publication, to Executive Editor, $M M W R$ Series, Mailstop E-90, CDC, 1600 Clifton Rd., N.E., Atlanta, GA 30329-4027 or to mmwrq@cdc.gov.

All material in the MMWR Series is in the public domain and may be used and reprinted without permission; citation as to source, however, is appreciated.

Use of trade names and commercial sources is for identification only and does not imply endorsement by the U.S. Department of Health and Human Services.

References to non-CDC sites on the Internet are provided as a service to $M M W R$ readers and do not constitute or imply endorsement of these organizations or their programs by CDC or the U.S. Department of Health and Human Services. CDC is not responsible for the content of these sites. URL addresses listed in $M M W R$ were current as of the date of publication.

ISSN: 1546-0738 (Print) 